\title{
Attractiveness of periodic orbits in parametrically forced systems with time-increasing friction
}

\author{
Michele Bartuccelli $^{1}$, Jonathan Deane ${ }^{1}$, Guido Gentile ${ }^{2}$ \\ ${ }^{1}$ Department of Mathematics, University of Surrey, Guildford, GU2 7XH, UK \\ 2 Dipartimento di Matematica, Università di Roma Tre, Roma, I-00146, Italy \\ E-mail: m.bartuccelli@surrey.ac.uk,j.deane@surrey.ac.uk, gentile@mat.uniroma3.it
}

\begin{abstract}
We consider dissipative one-dimensional systems subject to a periodic force and study numerically how a time-varying friction affects the dynamics. As a model system, particularly suited for numerical analysis, we investigate the driven cubic oscillator in the presence of friction. We find that, if the damping coefficient increases in time up to a final constant value, then the basins of attraction of the leading resonances are larger than they would have been if the coefficient had been fixed at that value since the beginning. From a quantitative point of view, the scenario depends both on the final value and the growth rate of the damping coefficient. The relevance of the results for the spin-orbit model are discussed in some detail.
\end{abstract}

\section{Introduction}

Take a one-dimensional system driven by an external force,

$$
\ddot{x}+F(x, t)=0
$$

with $F$ a smooth function $2 \pi$-periodic in time $t$; here and henceforth the dot denotes derivative with respect to time. Then add a friction term. Usually friction is modelled as a term proportional to the velocity, so that the equations of motion become

$$
\ddot{x}+F(x, t)+\gamma \dot{x}=0, \quad \gamma>0,
$$

with the proportionality constant $\gamma$ referred to as the damping coefficient.

As a consequence of friction attractors appear [33. If the system is a perturbation of an integrable one, there is strong evidence that all attractors are either equilibrium points (if any) or periodic orbits with periods $T$ which are rational multiples of the forcing period $2 \pi[5]$. If $2 \pi / T=p / q$, with $p, q \in \mathbb{N}$ and relatively prime, one says that the periodic orbit is a $p: q$ resonance. For each attractor one can study the corresponding basin of attraction, that is the set of initial data which approach the attractor as time goes to infinity. If all motions are bounded, one expects the union of all basins of attraction to fill the entire phase space, up to a set of zero measure. This appears to be confirmed by numerical simulations [19, 48, 7, [5].

Recently such a scenario has been numerically investigated in several models of physical interest, such as the dissipative standard map [19, 48, 20], the pendulum with oscillating suspension point [7], 
the driven cubic oscillator [5] and the spin-orbit model [2, 13. What emerges from the numerical simulations is that, for fixed damping, only a finite number of either point or periodic attractors is present and every initial datum in phase space is attracted by one of them, according to Palis' conjecture [39, 40]. However, which attractors are really present and the sizes of their basins depend on the value of the damping coefficient. If the latter is very small then many periodic attractors can coexist. This phenomenon is usually called multistability; see [19, 20]. By taking larger values for the damping coefficient, many of the attracting periodic orbits disappear and, eventually, when the coefficient becomes very large, only a few, if any, still persist: for every other resonance there is a threshold value for the damping coefficient above which the corresponding attractor disappears.

In this paper we aim to study what happens when the friction is not fixed but grows in time, more precisely when the damping coefficient is not a constant but a slowly increasing function of time. This is a very natural scenario: it is reasonable to suppose that in many physical contexts dissipation tends to increase to some asymptotic value. We aim to show (numerically) in such a setting, with the damping coefficient slowly increasing to a final value, that the relative areas of some basins of attraction become larger than they would be if the damping were fixed for all time at the final value. In other words, we claim that, in order to understand the dynamics of a forced system in the presence of damping, not only is the final value of the friction important, but also the time evolution of the damping itself plays a role. So, by looking in the present at a damped system which evolved from an original nearly conservative one, with the friction slowly increasing from virtually zero to the present value, it can happen that an attractor, which should have a small basin of attraction on the basis of the final value of the friction, is instead much larger than expected. Of course, as we shall see, several elements come into the picture, in particular the growth rate of the damping coefficient and the closeness between its threshold and final values.

We shall investigate in detail a model system, the driven cubic oscillator in the presence of friction, which is particularly suited for numerical investigations because of its simplicity. We shall first study in Section 2 some properties in the case of constant friction, with some details worked out in Appendix A. Then in Section 3 we shall see how the behaviour of the system is affected by the presence of a non-constant, in fact slowly increasing friction. In Section 4 we shall introduce another system of physical interest, the spin-orbit system, with some details deferred to Appendices B to E, and we shall discuss how the results described in the previous sections may be relevant to the study of its behaviour. Further comments are deferred to Section 5. Finally in Section 6 we draw our conclusions and briefly discuss open problems. Some discussion of the codes we used for the numerical analysis is given in Appendix F

\section{The driven cubic oscillator with constant friction}

Let us consider the cubic oscillator, subject to periodic forcing and in the presence of friction,

$$
\ddot{x}+x^{3}+\varepsilon f(t) x^{3}+\gamma \dot{x}=0, \quad f(t)=\cos t,
$$

where $x \in \mathbb{R}$ and $\varepsilon$ is a real parameter, called the perturbation parameter, that we shall suppose positive (for definiteness). Of course one could consider more general expressions for $f$ and the choice made here is for simplicity. The system (2.1) has been investigated in [5], with $\gamma$ a fixed positive constant. The constants $\varepsilon$ and $\gamma$ are two control parameters, measuring respectively the forcing and the dissipation of the system. 
We shall look at (2.1) as a non-autonomous first order differential equation, so that the phase space is $\mathbb{R}^{2}$. Note that $(x, \dot{x})=(0,0)$ is an equilibrium point for all values of $\varepsilon$ and $\gamma$. Moreover, for $\varepsilon=\gamma=0$ the system is integrable and all motions are periodic. One can write the solutions explicitly in terms of elliptic integrals [25, 5]. For $\varepsilon \neq 0$ (hence $\varepsilon>0$ ) and $\gamma>0$ fixed, a finite number of periodic orbits of the unperturbed system persist and, together with the equilibrium point, they attract every trajectory in phase space [5]. Such periodic orbits are called subharmonic solutions in the literature [26]. Each periodic orbit can be identified through the corresponding frequency or, better, the ratio $\omega:=p / q$ between its frequency and the frequency of the forcing term. For each periodic orbit one can compute the corresponding threshold value $\gamma(\omega, \varepsilon)$ : if $\gamma>\gamma(\omega, \varepsilon)$ the orbit ceases to exist, while for $\gamma<\gamma(\omega, \varepsilon)$ the orbit is present, with a basin of attraction whose area depends on the actual value of $\gamma$. At fixed $\varepsilon$, one has

$$
\lim _{\max \{p, q\} \rightarrow \infty} \gamma(p / q, \varepsilon)=0
$$

Therefore, if we assume that all attractors different from the equilibrium point are periodic and no periodic attractors other than subharmonic solutions exist, then we find that at fixed $\varepsilon$ and $\gamma$ only a finite number of attractors exists. We note that the assumption above, even though we have no proof, is consistent with numerical findings [5].

The threshold value $\gamma(\omega, \varepsilon)$ depends smoothly on $\varepsilon$ [14, 26, 22]: for all $\omega \in \mathbb{Q}$ there exists $n(\omega) \in \mathbb{N}$ such that the corresponding threshold value is of the form $\gamma(\omega, \varepsilon)=C_{0}(\omega, \varepsilon) \varepsilon^{n(\omega)}$, with the constant $C_{0}(\omega, \varepsilon)$ nearly independent of $\varepsilon$ for $\varepsilon$ small; more precisely $C_{0}(\omega, \varepsilon)$ tends to a constant $C_{0}(\omega)$ as $\varepsilon$ goes to zero, so that we can consider it a constant for $\varepsilon$ small enough. Resonances are classified as follows: we refer to resonances with frequency $\omega$ such that $n(\omega)=1$ as primary, to resonances with frequency $\omega$ such that $n(\omega)=2$ as secondary, and so on [43, 5]. Of course such a classification makes sense only for $\varepsilon$ small enough. The primary resonances are the most important, in the sense that, at fixed small $\varepsilon$, for $\gamma$ large enough, only primary resonances are present; moreover, by decreasing the value of $\gamma$, although non-primary resonances appear, they have a small basin of attraction with respect to those of the primary ones. The threshold values of the leading attractors (that is the attractors with largest threshold values), in terms of the constants $C_{0}(\omega)$, were computed analytically in [5] and are reproduced in Tables 2.1 and 2.2. In particular the periodic attractors with frequency $1 / q$, with $q$ odd, appear in pairs [5]. The higher order corrections to $C_{0}(\omega, \varepsilon)$ are explicitly computable; however we shall not need to do this here. Note that the classification of resonances and the corresponding threshold values strongly depend on the forcing: all values in this and next Section refer to $f(t)=\cos t$, as in 2.1 .

Table 2.1: Values of the constants $C_{0}(p / q)$ for $p=1$ and $q=2,4,6,8,10$ (leading primary resonances) for the cubic oscillator 2.1]; the threshold values are of the form $\gamma(\omega, \varepsilon)=C_{0}(\omega) \varepsilon+O\left(\varepsilon^{2}\right)$.

\begin{tabular}{|l|l|l|l|l|l|}
\hline \hline$q$ & 2 & 4 & 6 & 8 & 10 \\
\hline \hline$C_{0}(1 / q)$ & 0.178442 & 0.061574 & 0.008980 & 0.000920 & 0.000078 \\
\hline
\end{tabular}

Consider the system (2.1) at fixed $\varepsilon$. For $\gamma$ large enough, the only attractor left is the equilibrium point; in that case all trajectories eventually go toward this point, which becomes a global attractor (see Appendix A. If $\gamma$ is not too large — that is, according to Table 2.1, if $\gamma<C_{0}(1 / 2) \varepsilon$, up to 
Table 2.2: Values of the constants $C_{0}(p / q)$ for $p=1$ and $q=1,3,5,7,9$ (leading secondary resonances) for the cubic oscillator 2.1); the threshold values are of the form $\gamma(\omega, \varepsilon)=C_{0}(\omega) \varepsilon^{2}+O\left(\varepsilon^{3}\right)$.

\begin{tabular}{|l|l|l|l|l|l|}
\hline \hline$q$ & 1 & 3 & 5 & 7 & 9 \\
\hline$C_{0}(1 / q)$ & 0.146322 & 0.065001 & 0.006488 & 0.000177 & 0.000002 \\
\hline
\end{tabular}

higher order corrections - then, besides the equilibrium point, there is a finite number of other attractors, which are periodic orbits.

For $\varepsilon=0.1$, from Tables 2.1 and 2.2 one obtains the threshold values $\gamma(1 / 2,0.1) \approx 0.018$, $\gamma(1 / 4,0.1) \approx 0.0062, \gamma(1,0.1) \approx 0.0015, \gamma(1 / 6,0.1) \approx 0.00090, \gamma(1 / 3,0.1) \approx 0.00065, \gamma(1 / 8,0.1) \approx$ $0.000092, \gamma(1 / 5,0.1) \approx 0.000065$, and so on. Take the initial data in a finite domain of phase space, say the square $\mathcal{Q}=[-1,1] \times[-1,1]$ : the relative areas of the parts of the basins of attraction contained in $\mathcal{Q}$ for some values of $\gamma$ are given in Table 2.3. In principle the relative areas depend on the domain, but one expects that they do not change too much by changing the domain, provided the latter is not too small. Note that for $\varepsilon=0.1$ and $\gamma \leq 0.00005$, other attractors than those listed in Table 2.3 appear (namely periodic orbits with frequencies $1 / 8,1 / 5$ and $3 / 4$ for $\gamma=0.00005$; with frequencies $1 / 8,1 / 5,3 / 10,2 / 5,5 / 12$ and $3 / 4$ for $\gamma=0.00001$; and with frequencies $1 / 10,1 / 8,1 / 7$, $1 / 5,3 / 14,2 / 7,3 / 10,2 / 5,5 / 12,3 / 7,2 / 3$ and $3 / 4$ for $\gamma=0.000005)$, so explaining why the relative areas of the basins of attractions considered there do not sum up to $100 \%$. Small discrepancies for the other values are simply due to round-off error (the error on the data is in the first decimal digit; see Appendix F).

Table 2.3: Relative areas $A(\omega, \gamma), \%$, of the parts of the basins of attraction contained inside the square $\mathcal{Q}$ for $\varepsilon=0.1$ and some values of $\gamma$. The attractors are identified by the corresponding frequency ( 0 is the origin). The number of random initial conditions taken in $\mathcal{Q}$ is 1000000 up to $\gamma=0.0001,500000$ for $\gamma=0.00005$, 150000 for $\gamma=0.00001$ and 50000 for $\gamma=0.000005$.

\begin{tabular}{|l|c|c|c|c|c|c|c|c|c|}
\hline \hline$\omega$ & 0 & $1 / 2$ & $1 / 4$ & $1 \mathrm{a}$ & $1 \mathrm{~b}$ & $1 / 6$ & $1 / 3 \mathrm{a}$ & $1 / 3 \mathrm{~b}$ & $3 / 8$ \\
\hline \hline$\gamma=0.020000$ & 100.0 & 00.0 & 00.0 & 00.0 & 00.0 & 00.0 & 00.0 & 00.0 & 00.0 \\
\hline$\gamma=0.015000$ & 91.1 & 08.9 & 00.0 & 00.0 & 00.0 & 00.0 & 00.0 & 00.0 & 00.0 \\
\hline$\gamma=0.010000$ & 79.1 & 20.9 & 00.0 & 00.0 & 00.0 & 00.0 & 00.0 & 00.0 & 00.0 \\
\hline$\gamma=0.005000$ & 64.9 & 31.8 & 03.4 & 00.0 & 00.0 & 00.0 & 00.0 & 00.0 & 00.0 \\
\hline$\gamma=0.001000$ & 44.5 & 40.9 & 13.2 & 00.7 & 00.7 & 00.0 & 00.0 & 00.0 & 00.0 \\
\hline$\gamma=0.000500$ & 38.7 & 41.8 & 14.7 & 01.3 & 01.3 & 01.7 & 00.3 & 00.3 & 00.0 \\
\hline$\gamma=0.000100$ & 32.2 & 41.9 & 14.0 & 02.6 & 02.6 & 03.6 & 01.5 & 01.5 & 00.1 \\
\hline$\gamma=0.000050$ & 30.2 & 41.6 & 13.8 & 02.8 & 02.8 & 03.8 & 01.7 & 01.7 & 00.6 \\
\hline$\gamma=0.000010$ & 26.9 & 41.1 & 13.2 & 02.9 & 02.9 & 03.9 & 01.8 & 01.8 & 01.1 \\
\hline$\gamma=0.000005$ & 26.2 & 40.9 & 13.0 & 02.9 & 02.9 & 03.8 & 01.8 & 01.8 & 01.3 \\
\hline
\end{tabular}

If one plots the relative areas $A(\omega, \gamma)$ of the basins of attraction versus $\gamma$ one finds the situation depicted in Figure 2.1. Of course in general $A(\omega, \gamma)$ depends also on $\varepsilon$, i.e. $A(\omega, \gamma)=A(\omega, \gamma, \varepsilon)$, although we are not making explicit such a dependence since $\varepsilon$ has been fixed at $\varepsilon=0.1$; same comment applies to the quantities $A_{\max }(\omega)=A_{\max }(\omega, \varepsilon)$ and $A(\omega)=A(\omega, \varepsilon)$ to be introduced. 

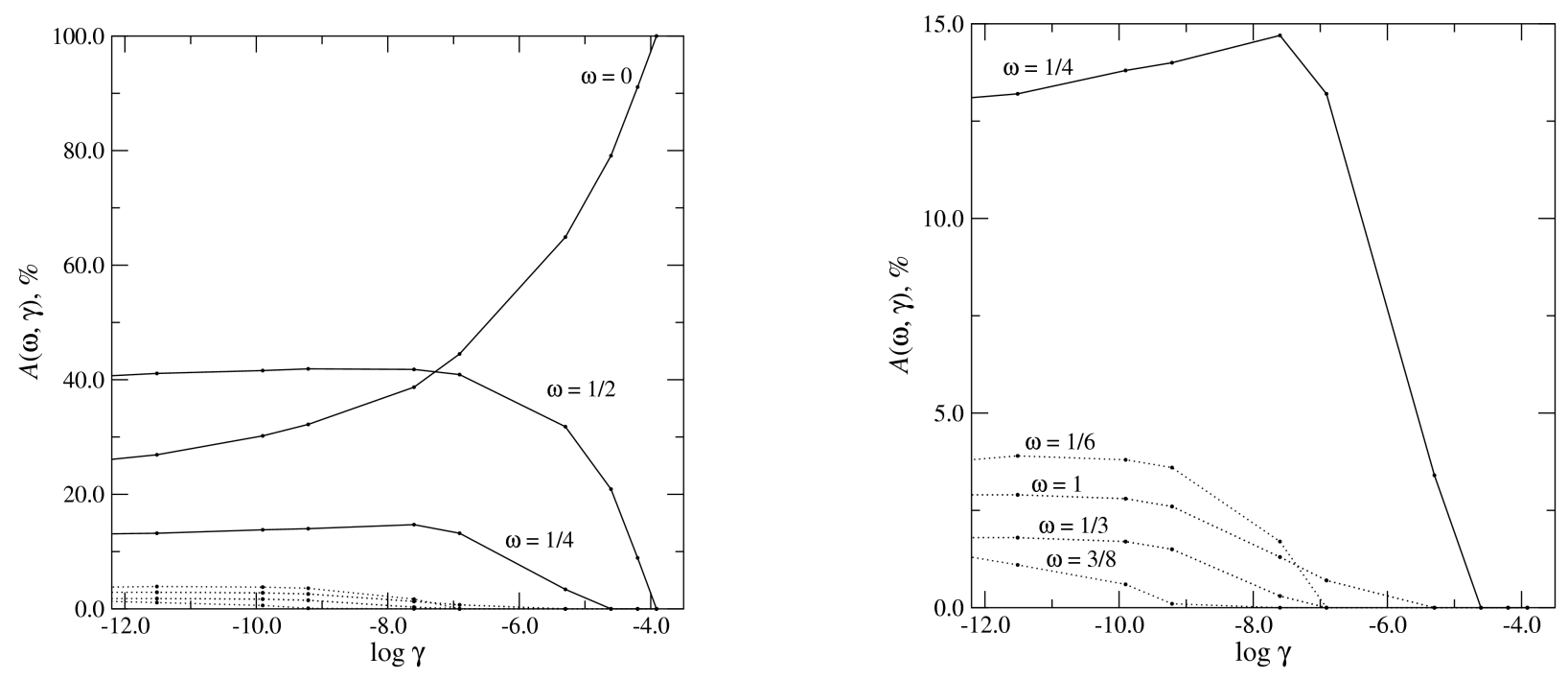

Figure 2.1: Relative areas $A(\omega, \gamma)$ of the basins of attraction versus $\log \gamma$ for the values of $\gamma$ listed in Table 2.3 (right-hand figure) and a magnification for the periodic orbits with $\omega=1 / 4,1,1 / 6,1 / 3,3 / 8$ (left-hand figure).

It can be seen that for any $\omega \in \mathbb{Q}$ one has $A(\omega, \gamma)=0$ if $\gamma>\gamma(\omega, \varepsilon)$. By decreasing $\gamma$ below $\gamma(\omega, \varepsilon), A(\omega, \gamma)$ increases up to a maximum value $A_{\max }(\omega)$ which tends to stabilise. For very small values of $\gamma$ one observes a slight bending downward. It would be interesting to investigate very - in principle arbitrarily - small values of $\gamma$, but of course we have to cope with the technical limitations of computation: studying arbitrarily small friction would require running programs for arbitrarily large times and with arbitrarily high precision — see also comments in Appendix F. However, by looking at Figure 2.1 and noting that numerical evidence suggests that all attractors different from the origin are periodic orbits, we make the following conjecture: as $\gamma$ goes to $0^{+}$, for all $\omega \in \mathbb{Q}$ the relative area $A(\omega, \gamma)$ tends to a finite limit $A(\omega)$ such that

$$
\sum_{\omega \in \mathbb{Q}} A(\omega)=100 \%
$$

where $\omega=0$ designates the origin. Of course when $\gamma=0$ the area of each basin drops to zero, so that, accepting the conjecture above, all functions $A(\omega, \gamma)$ are discontinuous at $\gamma=0$. This is not surprising: a similar situation arises in the absence of forcing, where the only attractor is the origin, with a basin of attraction which passes abruptly from zero $(\gamma=0)$ to $100 \%(\gamma>0)$. Moreover, analogously to [48, we would expect that the total number of periodic attractors $N_{\mathrm{p}}$ grows as a power of $\gamma$ when $\gamma$ tends to 0 . This means that if the limits $A(\omega)$ vanished at least one function $A(\omega, \gamma)$ should be exponential in $\log \gamma$, a behaviour which seems unlikely in the light of Figure 2.1.

\section{The driven cubic oscillator with increasing friction}

Here we shall consider $\gamma=\gamma(t)$ explicitly depending on time, that is

$$
\ddot{x}+x^{3}+\varepsilon f(t) x^{3}+\gamma(t) \dot{x}=0, \quad f(t)=\cos t,
$$


For both concreteness and simplicity reasons, we shall consider a dissipation $\gamma(t)$ linearly increasing in time up to some final value, i.e

$$
\gamma(t)= \begin{cases}\gamma_{0} \frac{t}{T_{0}}, & 0 \leq t<T_{0}, \\ \gamma_{0}, & t \geq T_{0}\end{cases}
$$

where the parameters $\gamma_{0}$ and $T_{0}$ are positive constants. However, the results we are going to describe should not depend too much on the exact form of the function $\gamma(t)$, as long as it is a slowly increasing function; see Section 5. In $(3.2)$ we shall take $T_{0}=\Delta / \gamma_{0}$, whose form is suggested by the fact that trajectories converge toward an attractor at a rate proportional to $1 / \gamma_{0}$ (see Appendix A).

Hence, consider the system with $\varepsilon=0.1$ again but now with $\gamma(t)$ given by $(3.2)$, with $T_{0}=\Delta / \gamma_{0}$ and $\gamma_{0}=0.015$. Computing the corresponding relative areas $A\left(\omega, \gamma_{0} ; \Delta\right)=A\left(\omega, \gamma_{0}, 0.1 ; \Delta\right)$ — that is $A\left(\omega, \gamma_{0}, \varepsilon ; \Delta\right)$ for $\varepsilon=0.1$ - for different values of $\Delta$, we obtain the results in Table 3.1 and Figure 3.1. If $\Delta$ is very small, the damping coefficient reaches the asymptotic value $\gamma_{0}$ almost immediately, and we would expect to obtain the same scenario as in the previous case ( $\gamma$ constant): two attractors, corresponding to the origin and the 1:2 resonance, with basins whose relative areas are close to the values for $\Delta=0$, i.e. $91.1 \%$ and $8.9 \%$, respectively. On the other hand, if $\Delta$ becomes larger, we find that the relative area of the basin of attraction of the origin decreases, whereas that of the basin of attraction of the 1:2 resonance increases. For $\Delta$ very large, these areas apparently tend to constant values of around $61 \%$ and $39 \%$, respectively; see Table 3.1 .

Table 3.1: Relative areas $A(\omega, 0.015 ; \Delta)$ of the parts of the basins of attraction contained inside the square $\mathcal{Q}$ for $\varepsilon=0.1$ and $\gamma(t)$ given by (3.2) with $\gamma_{0}=0.015$ and $T_{0}=\Delta / \gamma_{0}$, for various values of $\Delta$ and $\omega=0,1 / 2$ ( $\omega=0$ is the origin). In each case, 1000000 random initial conditions have been taken in $\mathcal{Q}$.

\begin{tabular}{|l|l|l|l|l|l|l|l|l|c|}
\hline \hline$\Delta$ & 0 & 25 & 50 & 75 & 100 & 125 & 150 & 175 & 200 \\
\hline \hline$\omega=0$ & 91.1 & 70.6 & 66.2 & 64.6 & 63.4 & 62.6 & 62.1 & 61.6 & 61.3 \\
\hline$\omega=1 / 2$ & 08.9 & 29.4 & 33.8 & 35.4 & 36.6 & 37.4 & 37.9 & 38.4 & 38.7 \\
\hline
\end{tabular}

Analogous results are found, for instance, for $\gamma_{0}=0.005$; see Table 3.2 and Figure 3.2 . One sees that for $\Delta=20$ the relative areas of the basins of attraction of the origin and of the 1:2 and 1:4 resonances have already appreciably changed: they have become, respectively, $53.4 \%, 38.5 \%$ and 8.1\%. By further increasing $\Delta$, once again a saturation phenomenon is observed and the relative areas settle about asymptotic values around $45 \%, 42 \%$ and $13 \%$ (for instance for $\Delta=120$ the areas are, respectively, $46.0 \%, 41.3 \%$ and $12.7 \%)$. Note that the value $\gamma_{0}=0.005$ is such that the threshold values $\gamma(1 / 2,0.1) \approx 0.018$ and $\gamma(1 / 4,0.1) \approx 0.0062$ of the persisting resonances are slightly above it (that is their ratios with $\gamma_{0}$ are of order 1 ).

If one fixes the value $\gamma_{0}=0.0005$, then the $1: 6,1: 1$ and 1:3 resonances are also present. On the other hand the threshold values of the 1:2 and 1:4 resonances are appreciably larger then $\gamma_{0}$ (that is $\gamma(\omega) \gg \gamma_{0}$ for $\omega=1 / 2$ and $\omega=1 / 4$ ), whereas the threshold values $\gamma(1 / 6,0.1) \approx 0.00090$, $\gamma(1,0.1) \approx 0.0015$ and $\gamma(1 / 3,0.1) \approx 0.00065$ of the $1: 6,1: 1$ and $1: 3$ resonances, respectively, are not too different from $\gamma_{0}$. If we again take $\gamma(t)$ as in $(3.2)$, with $T_{0}=\Delta / \gamma_{0}$ and $\gamma_{0}=0.0005$, we have the results in Table 3.3 and Figure 3.3.

Therefore we have, from a qualitative point of view, the same scenario as in the case $\gamma_{0}=0.005$, 


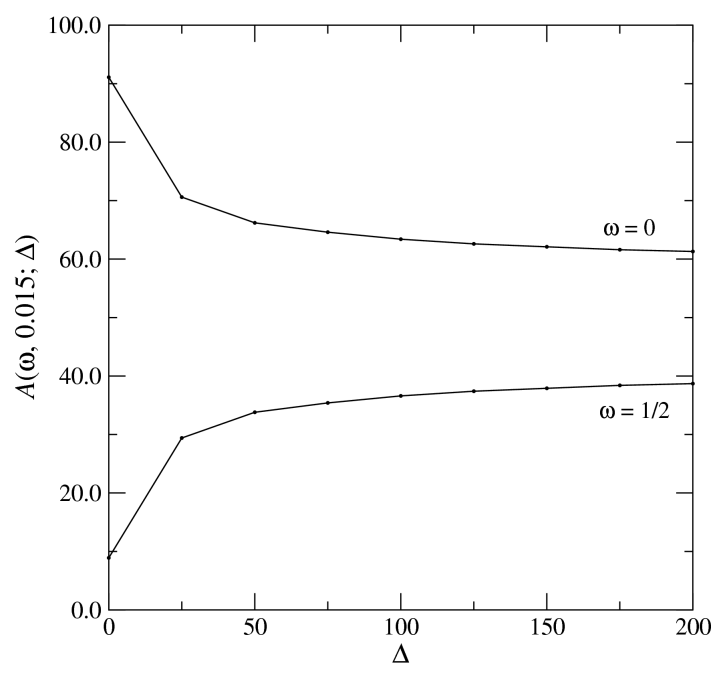

Figure 3.1: Relative measures of the basins of attraction versus $\Delta$ for $\gamma_{0}=0.015$.

Table 3.2: Relative areas $A(\omega, 0.005 ; \Delta)$ of the parts of the basins of attraction contained inside the square $\mathcal{Q}$ for $\varepsilon=0.1$ and $\gamma(t)$ given by $(3.2)$ with $\gamma_{0}=0.005$ and $T_{0}=\Delta / \gamma_{0}$, for various values of $\Delta$ and $\omega=0,1 / 2,1 / 4$ ( $\omega=0$ is the origin). 500000 random initial conditions have been taken in $\mathcal{Q}$.

\begin{tabular}{|l|l|l|l|l|l|l|l|}
\hline \hline$\Delta$ & 0 & 20 & 40 & 60 & 80 & 100 & 120 \\
\hline \hline$\omega=0$ & 64.8 & 53.4 & 49.4 & 47.9 & 46.8 & 46.1 & 46.0 \\
\hline$\omega=1 / 2$ & 31.8 & 38.5 & 40.0 & 40.7 & 41.2 & 41.5 & 41.3 \\
\hline$\omega=1 / 4$ & 03.4 & 08.1 & 10.6 & 11.4 & 12.0 & 12.4 & 12.7 \\
\hline
\end{tabular}

but with some relevant quantitative differences: the relative areas of the basins of the 1:2 and 1:4 resonances are not too different in the two situations $\gamma$ constant and $\gamma$ increasing.

We now give an argument to explain why the basins of attraction are different if $\gamma$ is not fixed ab initio to some value $\gamma_{0}$ but slowly tends to that value. According to Table 2.3, for smaller values of $\gamma$ the basins of the periodic attractors are larger. For instance for $\gamma=0.005$ the 1:2 and 1:4 resonances have basins with relative areas $31.8 \%$ and $3.4 \%$, respectively, while the basins of attraction of the same resonances for $\gamma=0.0005$ have relative areas $41.8 \%$ and $14.7 \%$, respectively. Then, if we suppose that the friction is slowly increasing in time, when it passes, say, from $\gamma=0.0005$ to 0.005 , on the one hand the size of the basin would decrease because of the larger value of $\gamma$, but on the other hand many trajectories have already nearly reached the basin and hence continue to be attracted toward that resonance. If friction increases slowly enough we can assume that it is quasi-static. Therefore, at every instant $\tau$, the basin of attraction of any resonance has the size corresponding to the value $\gamma(\tau)$ at that instant, as can be deduced by interpolation from Table 2.3 (or Figure ??), while the rate of approach to the resonance can be roughly estimated as proportional to $1 / \gamma(\tau)$; see Appendix A. Therefore if $\Delta$ is large enough (that is if the growth of $\gamma(t)$ is slow enough) one expects the trajectory to be captured by the resonance faster than how the basin of attraction is decreasing.

By increasing the friction further, the basin of a resonance $\omega$ can become negligible, until the resonance itself disappears. If this does not happen, that is if $\gamma(\omega, 0.1)>\gamma_{0}$, then there is a value 


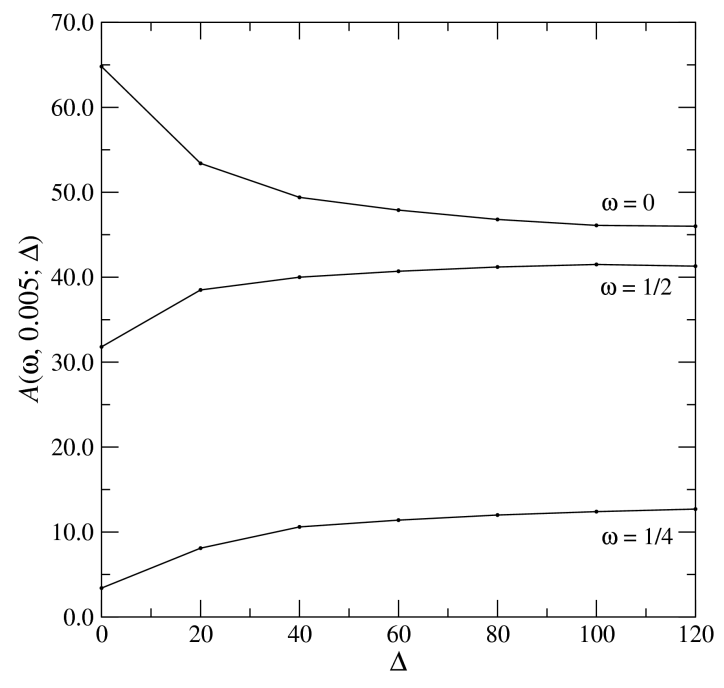

Figure 3.2: Relative areas of the basins of attraction versus $\Delta$ for $\gamma_{0}=0.005$.

Table 3.3: Relative areas $A(\omega, 0.0005 ; \Delta)$ of the parts of the basins of attraction contained inside $\mathcal{Q}$ for $\varepsilon=0.1$ and $\gamma(t)$ given by $(3.2)$ with $\gamma_{0}=0.0005$ and $T_{0}=\Delta / \gamma_{0}$, for various values of $\Delta$ and $\omega=0,1 / 2,1 / 4,1,1 / 6,1 / 3$ ( $\omega=0$ is the origin). 250000 random initial conditions have been taken in $\mathcal{Q}$.

\begin{tabular}{|l|l|l|l|l|l|l|l|l|l|}
\hline \hline$\Delta$ & 0 & 10 & 20 & 30 & 40 & 50 & 60 & 70 & 80 \\
\hline \hline$\omega=0$ & 38.7 & 36.4 & 34.8 & 34.1 & 33.5 & 33.3 & 33.1 & 32.9 & 32.7 \\
\hline$\omega=1 / 2$ & 41.8 & 40.9 & 41.4 & 41.6 & 41.5 & 41.5 & 41.6 & 41.3 & 41.6 \\
\hline$\omega=1 / 4$ & 14.7 & 13.9 & 13.8 & 13.6 & 13.9 & 13.9 & 13.8 & 14.0 & 13.9 \\
\hline$\omega=1$ & 01.3 & 02.9 & 02.9 & 02.9 & 02.9 & 03.0 & 03.0 & 03.0 & 03.0 \\
\hline$\omega=1 / 6$ & 01.6 & 01.8 & 02.4 & 02.9 & 02.9 & 03.0 & 03.2 & 03.1 & 03.2 \\
\hline$\omega=1 / 3$ & 00.3 & 00.6 & 00.9 & 01.0 & 01.2 & 01.2 & 01.3 & 01.4 & 01.3 \\
\hline
\end{tabular}

of $\Delta$ above which the relative measure $A\left(\omega, \gamma_{0} ; \Delta\right)$ of the basin saturates to a value close to the maximum value $A_{\max }(\omega)$ (possibly a bit smaller because of the slight bending downward observed in Figure 2.1). In particular, this explains the difference between Figures 3.2 and 3.3 . For concreteness let us focus on the 1:2 resonance. With respect to the case $\gamma_{0}=0.0005$, according to Figure 3.1 , the relative area $A(1 / 2, \gamma)$ of the basin of attraction does not increase appreciably when taking smaller values $\gamma<\gamma_{0}$ : indeed $A(1 / 2,0.0005)$ is already close to $A_{\max }(1 / 2)$.

We conclude that the main effect of friction slowly growing to a final value $\gamma_{0}$, is that eventually every basin of attraction has essentially the same size that would appear for lower values of friction. So, if the basin of attraction of any $p: q$ resonance is larger for values of friction lower than the final value, then, when the final value $\gamma_{0}$ is reached, one observes a basin of attraction with relative area larger than $A\left(p / q, \gamma_{0}\right)$. If on the contrary it is more or less the same, then one observes essentially the same basin one would have by taking the friction fixed at that value since the beginning. In other words, if the friction increases in time, one can really have a larger basin of attraction only if the final value $\gamma_{0}$ is close enough to the threshold value $\gamma(\omega, 0.1)$. However, if $\gamma_{0}$ is too close to $\gamma(\omega, 0.1)$, for the phenomenon to really occur, the rate of growth has to be slow enough: the closer $\gamma_{0}$ is to 


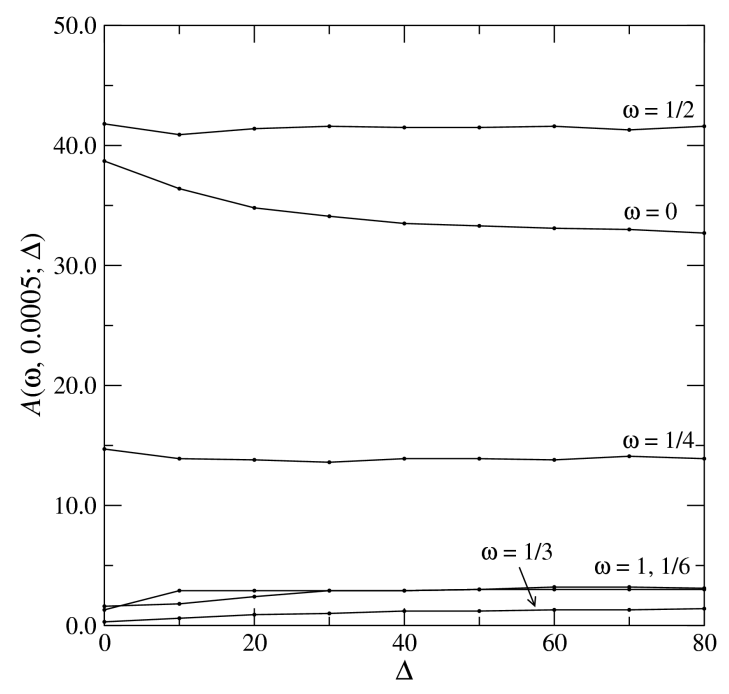

Figure 3.3: Relative areas of the basins of attraction versus $\Delta$ for $\gamma_{0}=0.0005$.

$\gamma(\omega, 0.1)$, the larger $\Delta$ to be chosen. For instance, for $\varepsilon=0.1$ and $\gamma_{0}=0.0005$, a glance at Table 3.3 gives the following picture. The areas of the basins of attraction for the 1:2 and 1:4 resonances have small variations for different values of $\Delta$, whereas the areas of the basins of attraction for 1:1, 1:3 and 1:6 change in a more appreciable way when $\Delta$ becomes larger. Moreover, the threshold value $\gamma(1 / 3,0.1) \approx 0.00065$ is just above $\gamma_{0}$, so for the area of the corresponding basin of attraction to come close to the maximum possible value one needs large values of $\Delta$; on the contrary $\gamma(1,0.1) \approx 0.00146$ is not too close to $\gamma_{0}$ and hence the area of the corresponding basin of attraction comes closer to the maximum possible value for smaller $\Delta \approx 10$.

We finish this section with a pair of figures showing the difference between the basins of attraction of the 1:2 resonance for $\gamma=0.005$, in the cases of constant and time-varying $\gamma$. According to Table 3.2 , changing $\Delta$ from zero - i.e. constant $\gamma$ - to 40 increases the area by about $8 \%$, and Figure 3.4 shows how this extra area is distributed (most of the points of the basin of attraction of the resonance for constant $\gamma$ still belong to the basin of attraction for varying $\gamma$ ).

\section{The spin-orbit model}

The spin-orbit model describes the motion of an asymmetric ellipsoidal celestial body (satellite) which moves in a Keplerian elliptic orbit around a central body (primary) and rotates around an axis orthogonal to the orbit plane [23, 36]. If $\theta$ denotes the angle between the longest axis of the satellite and the perihelion line, in the presence of tidal friction the model is described by the equation

$$
\ddot{\theta}+\varepsilon G(\theta, t)+\gamma(\dot{\theta}-1)=0,
$$

where $\varepsilon, \gamma>0$ and $\theta \in \mathbb{T}=\mathbb{R} / 2 \pi \mathbb{Z}$, so that the phase space is $\mathbb{T} \times \mathbb{R}$ (note that 4.1) is of the form $(1.2)$, with $x=\theta-t$ ). Here $\varepsilon$ is a small parameter, related to the asymmetry of the equatorial 

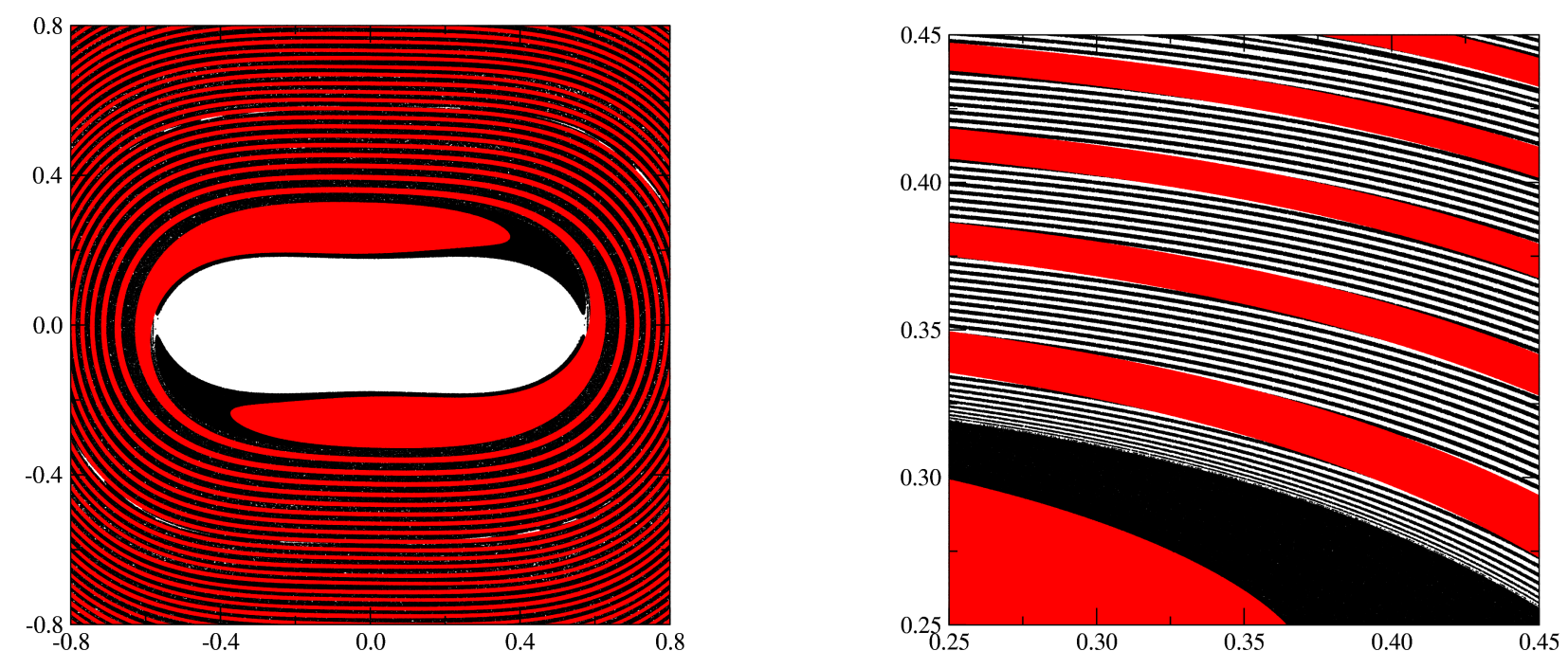

Figure 3.4: Basins of attraction for the 1:2 resonance for constant $\gamma$ (red) and time-varying $\gamma$ (black plus most of the red region). Initial conditions in the white region either go to the origin or to the 1:4 resonance. The right-hand figure shows a portion of the left-hand figure, magnified.

moments of inertia of the satellite, and $G(\theta, t)=\partial_{\theta} g(\theta, t)$, where

$$
\begin{aligned}
g(\theta, t) & =\left(\frac{1}{4} e-\frac{1}{32} e^{3}+\frac{5}{768} e^{5}\right) \cos (2 \theta-t)+\left(\frac{1}{2}-\frac{5}{4} e^{2}+\frac{13}{32} e^{4}\right) \cos (2 \theta-2 t) \\
& +\left(-\frac{7}{4} e+\frac{123}{32} e^{3}-\frac{489}{256} e^{5}\right) \cos (2 \theta-3 t)+\left(\frac{17}{4} e^{2}-\frac{115}{12} e^{4}\right) \cos (2 \theta-4 t) \\
& +\left(-\frac{845}{96} e^{3}+\frac{32525}{1536} e^{5}\right) \cos (2 \theta-5 t)+\left(\frac{533}{32} e^{4}\right) \cos (2 \theta-6 t) \\
& +\left(-\frac{228347}{7680} e^{5}\right) \cos (2 \theta-7 t)+\left(-\frac{1}{96} e^{3}-\frac{11}{1536} e^{5}\right) \cos (2 \theta+t) \\
& +\left(\frac{1}{48} e^{4}\right) \cos (2 \theta+2 t)+\left(-\frac{81}{2560} e^{5}\right) \cos (2 \theta+3 t),
\end{aligned}
$$

with $e$ being the eccentricity of the orbit; terms of order $O\left(e^{6}\right)$ have been neglected. In the celestial mechanics cases the model (4.1) may appear oversimplified and more realistic pictures could be devised [32, 15, 16. Nevertheless, because of its simplicity, it is suitable also for analytical investigations (as opposed to just numerical ones) on the relevance of friction in the early stages of evolution of celestial bodies and for the selection of structurally stable periodic motions.

Values of $e, \varepsilon$ and $\gamma$ for some primary-satellite systems of the solar system are given in Table 4.1. The values of $e$ can be found in the literature [36], while the derivation of $\varepsilon$ and $\gamma$ is discussed in Appendix B; see also below. All satellites of the solar system are trapped in the 1:1 resonance (rotation period equal to the revolution period), with the remarkable exception of Mercury (which can be considered as a satellite of the Sun), which turns out to be in a 3:2 resonance. The spin-orbit model has been used since the seminal paper by Goldreich and Peale 23] in an effort to explain the anomalous behaviour of Mercury. The ultimate reason is speculated to be related to the large value of the eccentricity. However, even though higher than for the other primary-satellite systems, the probability of capture into the 3:2 resonance is still found to be rather low [23, 13]. In the following 
part of this section we aim to investigate what happens if we take into account the fact that friction increased during the evolution history of the satellites.

Table 4.1: Values of the constants $e, \varepsilon$ and $\gamma$ for some cases of physical interest for the spin-orbit model (4.1).

\begin{tabular}{|l|l|l|l|l|l|}
\hline \hline & Primary & Satellite & $e$ & $\varepsilon$ & $\gamma$ \\
\hline \hline E-M & Earth & Moon & 0.0549 & $6.75 \times 10^{-7}$ & $3.75 \times 10^{-8}$ \\
\hline S-M & Sun & Mercury & 0.2056 & $8.11 \times 10^{-7}$ & $3.24 \times 10^{-8}$ \\
\hline J-G & Jupiter & Ganymede & 0.0013 & $4.30 \times 10^{-4}$ & $1.91 \times 10^{-5}$ \\
\hline J-I & Jupiter & Io & 0.0041 & $3.85 \times 10^{-3}$ & $1.71 \times 10^{-4}$ \\
\hline S-E & Saturn & Enceladus & 0.0047 & $1.41 \times 10^{-2}$ & $6.26 \times 10^{-4}$ \\
\hline S-D & Saturn & Dione & 0.0022 & $3.85 \times 10^{-3}$ & $1.71 \times 10^{-4}$ \\
\hline
\end{tabular}

Again one can compute the threshold values of the primary resonances, by writing $\gamma(\omega)=C_{0}(\omega) \varepsilon$, up to higher order corrections. If one writes 4.2 as

$$
g(\theta, t)=\sum_{k \in \mathbb{Z}} a_{k} \cos (2 \theta-k t)
$$

one finds (see Appendix D)

$$
C_{0}(p / q)=\frac{2 q\left|a_{2 p / q}\right|}{|p-q|}, \quad \frac{p}{q} \in\left\{-1, \pm \frac{1}{2}, \pm \frac{3}{2}, 2, \frac{5}{2}, 4, \frac{7}{2}\right\}
$$

while $C_{0}(1)=\infty$ (that is no threshold value exists for the $1: 1$ resonance) and $C_{0}(\omega)=0$ for any other $\omega$; other resonances may appear only at higher order in $\varepsilon$. This leads to the values listed in Table 4.2, for the primary resonances of the systems considered in Table 4.1. Note that for $\gamma$ large enough, all attractors disappear except the 1:1 resonance, which becomes a global attractor.

Table 4.2: Values of the constants $C_{0}(p / q)$ for some primary resonances of the the spin-orbit model 4.1); the threshold values are of the form $\gamma(\omega, \varepsilon)=C_{0}(\omega) \varepsilon$. Only positive $\omega$ have been explicitly considered.

\begin{tabular}{|l|l|l|l|l|l|l|l|}
\hline & $\omega$ & $1 / 2$ & $3 / 2$ & 2 & $5 / 2$ & 3 & $7 / 2$ \\
\hline \hline E-M & $C_{0}(\omega)$ & $5.488 \times 10^{-2}$ & $3.818 \times 10^{-1}$ & $2.545 \times 10^{-2}$ & $1.928 \times 10^{-3}$ & $1.513 \times 10^{-4}$ & $1.186 \times 10^{-5}$ \\
\hline S-M & $C_{0}(\omega)$ & $2.045 \times 10^{-1}$ & 1.308 & $3.251 \times 10^{-1}$ & $9.163 \times 10^{-2}$ & $2.976 \times 10^{-2}$ & $8.739 \times 10^{-3}$ \\
\hline J-G & $C_{0}(\omega)$ & $1.300 \times 10^{-3}$ & $9.100 \times 10^{-3}$ & $1.436 \times 10^{-5}$ & $2.578 \times 10^{-8}$ & $4.757 \times 10^{-11}$ & $8.832 \times 10^{-14}$ \\
\hline J-I & $C_{0}(\omega)$ & $4.100 \times 10^{-3}$ & $2.870 \times 10^{-2}$ & $1.429 \times 10^{-4}$ & $8.088 \times 10^{-7}$ & $4.707 \times 10^{-9}$ & $2.756 \times 10^{-11}$ \\
\hline S-E & $C_{0}(\omega)$ & $4.700 \times 10^{-3}$ & $3.290 \times 10^{-2}$ & $1.878 \times 10^{-4}$ & $1.218 \times 10^{-6}$ & $8.128 \times 10^{-9}$ & $5.455 \times 10^{-11}$ \\
\hline S-D & $C_{0}(\omega)$ & $2.200 \times 10^{-3}$ & $1.540 \times 10^{-2}$ & $4.114 \times 10^{-5}$ & $1.259 \times 10^{-7}$ & $3.902 \times 10^{-10}$ & $1.226 \times 10^{-12}$ \\
\hline
\end{tabular}

It seems reasonable on physical grounds (see Appendix E) to assume that friction was increasing in the past up to the present-day value $\gamma_{0}=\gamma$, with $\gamma$ as in Table 4.1. Application to the spin-orbit model for S-M would require numerics with very small values of $\varepsilon$ and $\gamma_{0}$ : the discussion in Appendix $\mathrm{B}$ provides the values in Table 4.1, so that $\gamma_{0} \sim 0.05 \varepsilon$. However, the value usually taken for $\varepsilon$ in the literature is $\varepsilon \sim 10^{-4}$ (see Appendix B. In both cases, $\gamma_{0}$ is far below the threshold value $\gamma(\omega, \varepsilon)$, 
especially for the most interesting resonance $\omega=3 / 2$ [23, 36], as $\gamma(3 / 2, \varepsilon) \sim 1.3 \varepsilon$ (see Table 4.2]; we refer to Section 6 for further comments.

As already noted in [13, the small value of $\gamma_{0}$ represents a serious difficulty from a numerical point of view, because it requires very long integration (for a very large number of initial conditions - see comments in Appendix F). Nevertheless, the discussion in Section 3 about the driven cubic oscillator allows us to draw the following conclusions about the spin-orbit model.

The results in Table 2.3 suggest that the relative areas $A(\omega, \gamma, \varepsilon)$ of the basins of attraction are almost constant for values of $\gamma$ much smaller than the threshold values; more precisely they assume values close to $A_{\max }(\omega, \varepsilon)$. In the case of increasing friction, if the final value $\gamma_{0}$ is much smaller than the threshold value, the basin turns out to have more or less the same size close to $A_{\max }(\omega, \varepsilon)$ as it would have if $\gamma$ were set equal to $\gamma_{0}$ since the beginning. The same scenario is expected in the case of the spin-orbit problem. In particular a crucial aspect is understanding when the final value can be considered 'much smaller' than the threshold value or comparable to it. Again the analysis in Section 3 is useful: pragmatically, we shall define $\gamma$ much smaller than $\gamma(\omega, \varepsilon)$ when $A(\omega, \gamma, \varepsilon)$ is close to the maximum possible value $A_{\max }(\omega, \varepsilon)$. Therefore it becomes fundamental to check whether, for the current values of $\gamma$ and $\varepsilon$ as given in the literature, $A(\omega, \gamma, \varepsilon)$ is either close to $A_{\max }(\omega, \varepsilon)$ or much smaller.

1. If we assumed $A(\omega, \gamma, \varepsilon)$ to be close to $A_{\max }(\omega, \varepsilon)$ the time-dependence of friction would not change the general picture as observed today. From this point of view, our results would be a bit disappointing: indeed the relative area of the basin of attraction of the $3: 2$ resonance, with the values of $\varepsilon$ and $\gamma$ usually taken in the literature, is found to be rather small for $\mathrm{S}-\mathrm{M}$ [13] and including the time-dependence of friction in the analysis would not give larger estimates. On the other hand, also in this second case, our analysis would provide some more information: it would yield that the results available in the literature [15, 16, 13] would remain correct even if time-dependent friction were included. In particular, to explain why Mercury has been captured into the 3:2 resonance, other mechanisms should be be invoked, such as the chaotic evolution of its orbit [15]. Of course the values of $\varepsilon$ and $\gamma$ used in the literature are only speculative: again our analysis suggests that the results would not change in a sensible way even by taking different values for one or both parameters - see also comments in Section 6 .

2. On the contrary if $A(\omega, \gamma, \varepsilon)$ were much smaller than $A_{\max }(\omega, \varepsilon)$, taking into account the timedependence of friction would imply a larger basin of attraction with respect to the case of constant friction. In this case the the exact values of the parameters $\varepsilon$ and $\gamma$ would play a fundamental role - again see also Section 6 .

\section{$5 \quad$ Remarks and comments}

\subsection{Different values of the perturbation parameter}

In Sections 2 and 3 we have fixed $\varepsilon=0.1$. However, by taking different values of $\varepsilon$, the phenomenology does not change. For instance, for $\varepsilon=0.5$ and $\varepsilon=0.01$ we have the relative areas listed in Tables 5.1 and 5.2 (for $\varepsilon=0.5$ only a few attractors are taken into account in Table 5.1).

The general scenario is the same as in the case $\varepsilon=0.1$, with obvious quantitative differences due to the fact that for smaller values of $\varepsilon$ (say $\varepsilon=0.01$ ) only primary resonances are relevant unless $\gamma$ is 
Table 5.1: Relative areas of the parts of the basins of attraction contained inside the square $\mathcal{Q}$ for $\varepsilon=0.5$. ( $\omega=0$ denotes the origin). 1000000 random initial conditions have been taken in $\mathcal{Q}$.

\begin{tabular}{|l|c|c|c|l|l|l|}
\hline \hline$\omega$ & 0 & $1 / 2$ & $1 / 4$ & $1 \mathrm{a}$ & $1 \mathrm{~b}$ & $1 / 6$ \\
\hline$\gamma=0.1000$ & 100.0 & 00.0 & 00.0 & 00.0 & 00.0 & 00.0 \\
\hline$\gamma=0.0750$ & 70.5 & 29.5 & 00.0 & 00.0 & 00.0 & 00.0 \\
\hline$\gamma=0.0500$ & 49.8 & 50.2 & 00.0 & 00.0 & 00.0 & 00.0 \\
\hline$\gamma=0.0250$ & 32.0 & 56.0 & 05.8 & 03.1 & 03.1 & 00.0 \\
\hline$\gamma=0.0050$ & 10.4 & 48.8 & 07.7 & 10.5 & 10.5 & 00.0 \\
\hline$\gamma=0.0025$ & 08.1 & 36.3 & 06.8 & 11.5 & 11.5 & 00.8 \\
\hline$\gamma=0.0010$ & 06.9 & 37.5 & 04.4 & 11.4 & 11.4 & 01.6 \\
\hline
\end{tabular}

Table 5.2: Relative areas of the parts of the basins of attraction contained inside the square $\mathcal{Q}$ for $\varepsilon=0.01$. ( $\omega=0$ denotes the origin). 500000 random initial conditions have been taken in $\mathcal{Q}$.

\begin{tabular}{|l|c|c|c|c|c|c|}
\hline \hline$\omega$ & 0 & $1 / 2$ & $1 / 4$ & $1 \mathrm{a}$ & $1 \mathrm{~b}$ & $1 / 6$ \\
\hline$\gamma=0.0020$ & 100.0 & 00.0 & 00.0 & 00.0 & 00.0 & 00.0 \\
\hline$\gamma=0.0015$ & 98.1 & 01.9 & 00.0 & 00.0 & 00.0 & 00.0 \\
\hline$\gamma=0.0010$ & 94.0 & 06.0 & 00.0 & 00.0 & 00.0 & 00.0 \\
\hline$\gamma=0.0005$ & 88.3 & 10.6 & 01.1 & 0.00 & 0.00 & 00.0 \\
\hline$\gamma=0.0001$ & 78.2 & 15.3 & 06.5 & 00.0 & 00.0 & 00.0 \\
\hline
\end{tabular}

very small, while for larger $\varepsilon$ (say $\varepsilon=0.5$ ) more and more resonances appear by taking smaller values of $\gamma$ (because powers of $\varepsilon$ are not much smaller than $\varepsilon$ itself). Indeed, if $\varepsilon=0.5$, even for $\gamma=0.005$ we detect numerically more than 50 attractors and the classification of resonance $\omega$ according to the the value of $n(\omega)$ becomes meaningless. For instance, for $\varepsilon=0.5$, one has $A(3 / 4,0.0025,0.5) \approx 11.4 \%$ and $A(3 / 4,0.001,0.5) \approx 6.8 \%$, to be compared with the values for $\omega=1 / 4$ in Table 5.1. Moreover for large values of $\varepsilon$, say for $\varepsilon=0.5$, the bending of the curves $A(\gamma, \omega, \varepsilon)$ in Figure ?? is more pronounced and the monotonic decrease observed for $\varepsilon=0.1$ when $\gamma$ tends to 0 seems to be violated (compare the values $A(1 / 2, \gamma, 0.5)$ for $\gamma=0.0025$ and $\gamma=0.001$ in Table 5.1); see also the comments in Section 6.

\subsection{Different functions $\gamma(t)$}

As stated at the beginning of Section 3 , the exact form of the function $\gamma(t)$ should not be relevant. As a check we studied (3.1) with $\gamma(t)$ given by both $(3.2)$ and

$$
\gamma(t)=\gamma_{0}\left(1-\mathrm{e}^{-t / T_{0}}\right)
$$

where $\gamma_{0}$ and $T_{0}$ are positive constants, by setting $T_{0}=\Delta / \gamma_{0}$ and changing $\Delta$ for fixed values of $\gamma_{0}$. The results show that the same behaviour is obtained in both cases. For instance, for $\gamma_{0}=0.006$, one has the results in Tables 5.3 and 5.4 see also Figure 5.1.

In particular, in both cases the relative areas $A\left(\omega, \gamma_{0}, 0.1 ; \Delta\right)$ start at the values corresponding to constant dissipation $\gamma=\gamma_{0}$ for $\Delta=0$ and then either decrease (for the origin) or increase (for the 
Table 5.3: Relative areas $A(\omega, 0.006,0.1 ; \Delta)$ of the parts of the basins of attraction contained inside the square $\mathcal{Q}$ for $\varepsilon=0.1$ and $\gamma(t)$ given by 3.2 with $\gamma_{0}=0.006$ and $T_{0}=\Delta / \gamma_{0}$, for various values of $\Delta$ and $\omega=0,1 / 2,1 / 4$. ( $\omega=0$ denotes the origin). 500000 random initial conditions have been taken in $\mathcal{Q}$.

\begin{tabular}{|l|l|l|l|l|l|l|}
\hline \hline$\Delta$ & 0 & 10 & 20 & 30 & 40 & 50 \\
\hline$\omega=0$ & 69.6 & 64.3 & 56.3 & 53.1 & 51.4 & 50.1 \\
\hline$\omega=1 / 2$ & 29.9 & 34.8 & 37.2 & 38.6 & 39.2 & 39.8 \\
\hline$\omega=1 / 4$ & 00.5 & 00.9 & 06.5 & 08.3 & 09.4 & 10.1 \\
\hline
\end{tabular}

Table 5.4: Relative areas $A(\omega, 0.006,0.1 ; \Delta)$ of the parts of the basins of attraction contained inside the square $\mathcal{Q}$ for $\varepsilon=0.1$ and $\gamma(t)$ given by $\left[5.1\right.$ with $\gamma_{0}=0.006$ and $T_{0}=\Delta / \gamma_{0}$, for various values of $\Delta$ and $\omega=0,1 / 2,1 / 4$ $(\omega=0$ denotes the origin). 500000 random initial conditions have been taken in $\mathcal{Q}$.

\begin{tabular}{|l|l|l|l|l|l|l|l|l|}
\hline \hline$\Delta$ & 0 & 1 & 2 & 8 & 13 & 20 & 30 & 40 \\
\hline \hline$\omega=0$ & 69.6 & 69.3 & 67.0 & 58.3 & 55.8 & 53.3 & 51.3 & 50.0 \\
\hline$\omega=1 / 2$ & 29.9 & 30.1 & 31.8 & 36.1 & 37.1 & 38.2 & 39.1 & 39.7 \\
\hline$\omega=1 / 4$ & 00.5 & 00.6 & 01.2 & 05.6 & 07.2 & 08.5 & 09.6 & 10.3 \\
\hline
\end{tabular}

periodic orbits), apparently to some asymptotic value close to $A_{\max }(\omega, 0.1)$. For instance the relative areas corresponding to (3.2) with $\Delta=30$ are very close to those corresponding to (5.1) with $\Delta=20$ (compare Table 5.3 with Table 5.4). The asymptotic values seem to be the same in both cases.

\section{Conclusions and open problems}

In this paper we have studied how the slow growth of friction may affect the asymptotic behaviour of dissipative dynamical systems. We have focused on a simple paradigmatic model, the periodically driven cubic oscillator, particularly suited for numerical investigations. Nevertheless we think that the results hold in the more general setting considered in Section11. The main result, discussed in Section 3. can be summarised as follows: on the one hand it is the final value of the damping coefficient that determines which attractors are present, but on the other hand the sizes of the corresponding basins of attraction strongly depend on the full evolution of the damping coefficient itself, in particular on its growth rate. Let $\gamma(p / q, \varepsilon)$ and $\gamma_{0}$ denote the threshold value of the $p: q$ resonance and the final value of the damping coefficient, respectively. If $\gamma_{0}>\gamma(p / q, \varepsilon)$ the attractor disappears. Otherwise the following possibilities arise: if $\gamma_{0} \ll \gamma(p / q, \varepsilon)$ the area of the corresponding basin of attraction is more or less the same as in the case with constant damping coefficient equal to $\gamma_{0}$, whereas it is larger if $\gamma_{0} \lesssim \gamma(p / q, \varepsilon)$. In the latter case, the closer $\gamma_{0}$ is to $\gamma(p / q, \varepsilon)$, the slower the growth rate of $\gamma(t)$ required for the maximum possible area of the basin of attraction to be attained. Moreover when $\gamma_{0} \ll \gamma(p / q, \varepsilon)$ the area can be even a little smaller than what it would be if the damping were fixed at $\gamma_{0}$ since $t=0$, because other attractors may have acquired a larger basin of attraction while the damping coefficient increased. It is not possible for the relative area to be larger than the value $A_{\max }(p / q, \varepsilon)$, which therefore represents an upper bound.

Finally, let us mention a few open problems which would deserve further investigation, also in 

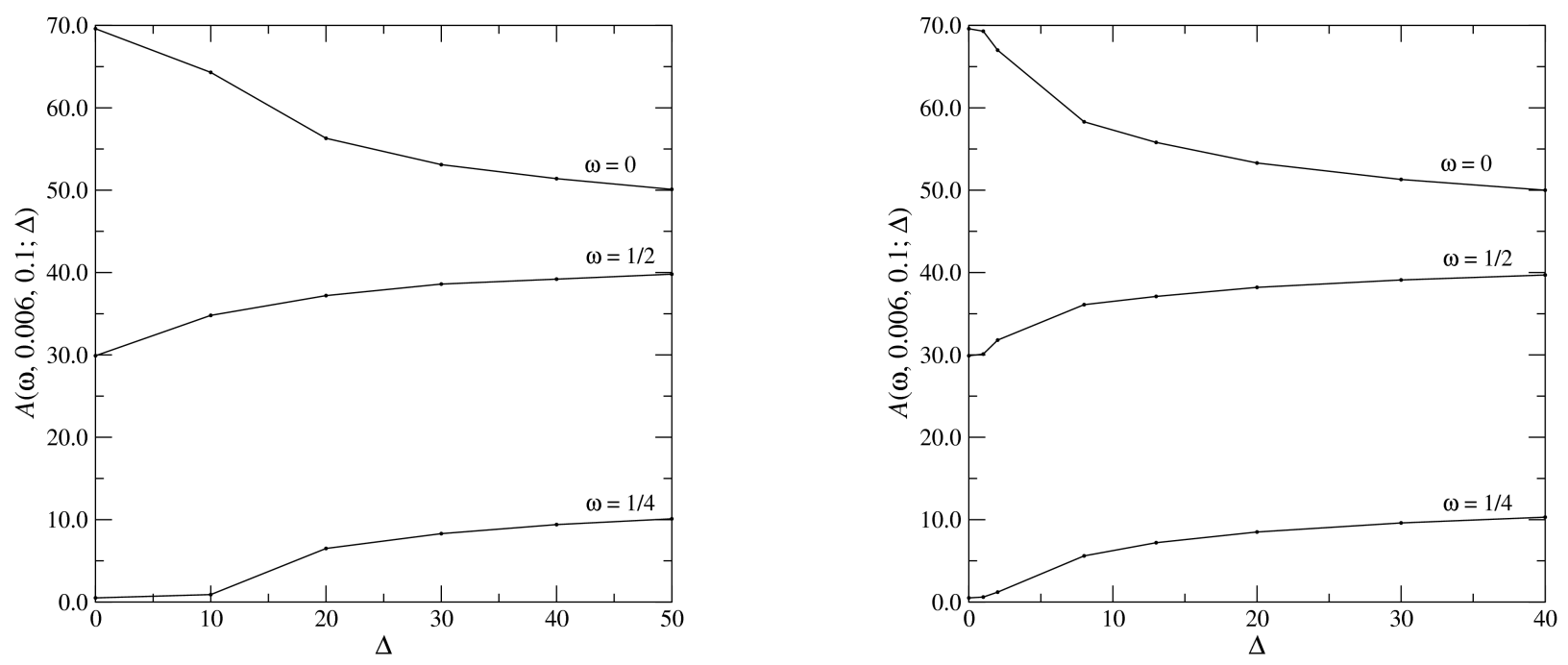

Figure 5.1: Relative areas of the basins of attraction versus $\Delta$ for $\gamma(t)$ given by 3.2 , with $\gamma_{0}=0.006$ (left-hand figure) and for $\gamma(t)$ given by (5.1), with $\gamma_{0}=0.006$ (right-hand figure).

relation with the spin-orbit problem.

1. As far as a dynamical system can be considered as a perturbation of an integrable one, all attractors seem to be either equilibrium points or periodic orbits: at least, this is what emerges from numerical simulations. It would be interesting to have a proof of this behaviour, even for some simple model such as (2.1), in particular of the fact that neither strange attractors nor periodic solutions other than subharmonic ones appear.

2. The analysis presented in Section 3 covers small values of $\gamma$, but still not as small as desirable. It would be worthwhile to study the behaviour of the curves $A(\omega, \gamma, \varepsilon)$ in the limit of even smaller $\gamma$, for instance by implementing some numerical integrator which allows us to decrease the running time of the programs without losing accuracy in the results.

3. In studying the spin-orbit model in Section 4 if one really wanted to consider the past history of the system, then not only $\gamma$ but also $\varepsilon$ should be taken to depend on time: this would introduce further difficulties and a more detailed model for the evolution of the satellite would be needed (see also comments at the end of Appendix B). Of course, this would be by no means an easy task. The use of a model for the time-dependence of friction already raises several problems, as we highlight in Appendix E.

4. We have seen that, for the spin-orbit model, in the case of constant friction the exact value of the parameters $\varepsilon$ and $\gamma$ is fundamental. For instance if $\gamma=\gamma_{1}$ such that $A\left(\omega, \gamma_{1}, \varepsilon\right) \approx A_{\max }(\omega, \varepsilon)$, then we have a basin of attraction much larger than for $\gamma=\gamma_{2}$, where $\gamma_{2} \gg \gamma_{1}$ is close to the threshold value $\gamma(\omega, \varepsilon)$. On the contrary, our analysis shows that, when one takes into account that friction slowly increased during the solidification process of the satellite, then for both values $\gamma_{1}$ and $\gamma_{2}$ we expect more or less the same relative area close to $A_{\max }(\omega, \varepsilon)$. This is useful information because it shows that exact values of the parameters $\varepsilon$ and $\gamma$ are 
not essential in the case of time-dependent friction, as long as $\gamma$ is not much smaller than the threshold value (we mean 'much smaller' in the sense of Section 44): of course the values of $\gamma$ and $\varepsilon$ are essential if $\gamma$ is much smaller than $\gamma(\omega, \varepsilon)$ - see next item.

5. As remarked in Section 4, to study the probability of capture of Mercury into the 3:2 resonance, it becomes crucial to determine the value $A_{\max }(3 / 2, \varepsilon)$ and the current values of $\gamma$ and $\varepsilon$ to see if $\gamma$ turns out to be much smaller than $\gamma(3 / 2, \varepsilon)$, that is if one has $A(3 / 2, \gamma) \ll A_{\max }(3 / 2, \varepsilon)$. If this were the case, by using time-dependent friction, the relative area of the basin of attraction of the 3:2 resonance would be much larger than what was found for constant damping coefficient $\gamma$ (estimated around 13\% in [13]). By assuming the values of $\gamma$ given in the literature, for which $\gamma \sim 10^{-8}$ and $\varepsilon \sim 10^{-4}$, and using that $\gamma(3 / 2, \varepsilon) \sim 1.3 \varepsilon$, the relation $A(3 / 2, \gamma) \ll A_{\max }(3 / 2, \varepsilon)$ could be verified only by assuming for $A(3 / 2, \gamma, \varepsilon)$ a very slow variation in $\gamma$ for $\varepsilon \sim 10^{-4}$. This does not seem impossible: already for the driven cubic oscillator the profiles of the curves $A(\omega, \gamma, \varepsilon)$ seem to have a much smoother variation for smaller values of $\varepsilon$ (compare Table 2.3 for $\varepsilon=0.1$ with Table 5.1 for $\varepsilon=0.5$ ), so it could happen then by decreasing $\varepsilon$ further the curves $A(\omega, \gamma, \varepsilon)$ could be nearly flat on much longer intervals: in other words, by fixing $\varepsilon \sim 10^{-4}$ and decreasing $\gamma$, the curve $A(\omega, \gamma, \varepsilon)$ could have not yet reached its maximum value $A_{\max }(\omega, \varepsilon)$ at $\gamma \sim 10^{-8}$. As a consequence, the exact values of the parameters $\varepsilon$ and $\gamma$ and the profile of the curve $A(3 / 2, \varepsilon, \gamma)$ could be fundamental. In any case, it would be very interesting to study numerically the spin-orbit model for very small values of $\varepsilon$ and $\gamma$, in order to obtain the profiles of the curves $A(3 / 2, \gamma, \varepsilon)$ versus $\gamma$.

Acknowledgments. We are grateful to Giovanni Gallavotti for very useful discussions and critical remarks, especially on the spin-orbit model and the formation and evolution of celestial bodies.

\section{A Some analytical results on system (2.1)}

\section{A.1 Global attraction to the origin for large $\gamma$}

For $\varepsilon$ small enough introduce the positive function $F(t)$ such that $F^{2}(t)=1+\varepsilon f(t)$ and rescale time through the Liouville transformation [35, 6]

$$
\tau:=\int_{0}^{t} \mathrm{~d} s F(s)
$$

Then we can rewrite (2.1) as

$$
\left\{\begin{array}{l}
x^{\prime}=y, \\
y^{\prime}=-x^{3}-\frac{y}{F(t)}\left(\gamma+\frac{F^{\prime}(t)}{F(t)}\right),
\end{array}\right.
$$

where the prime denotes derivative with respect to $\tau$. Define

$$
I(x, \dot{x}, t):=\frac{y^{2}}{2}+\frac{x^{4}}{4}
$$


which is an invariant for (2.1) with $\varepsilon=\gamma=0$. More generally one has

$$
I^{\prime}=-\frac{1}{F(t)}\left(\gamma+\frac{\varepsilon \dot{f}(t)}{2(1+\varepsilon f(t))}\right) y^{2},
$$

so that, if

$$
\gamma>-\min \frac{\varepsilon \dot{f}(t)}{2(1+\varepsilon f(t))}
$$

by using Barbashin-Krasovsky-La Salle's theorem [31, we find that the origin is an asymptotically stable equilibrium point and every initial datum is attracted to it as $t \rightarrow \infty$.

\section{A.2 Rate of convergence to attractors}

The periodic orbits for the system (2.1) appear in pairs of stable and unstable orbits: this is a consequence of Poincaré-Birkhoff's theorem [9]. Let us consider the primary resonances, so that we can set $\gamma=\varepsilon C$, with $C$ a constant independent of $\varepsilon$.

We rewrite the equations of motion in action-angle variables $(I, \varphi)[\underline{5}$

$$
\left\{\begin{array}{l}
\dot{\varphi}=(3 I)^{1 / 3}+\varepsilon(3 I)^{1 / 3} f(t) \operatorname{cn}^{4} \varphi-\varepsilon C \operatorname{cn} \varphi \operatorname{sn} \varphi \operatorname{dn} \varphi \\
\dot{I}=\varepsilon(3 I)^{4 / 3} f(t) \operatorname{cn}^{3} \varphi \operatorname{sn} \varphi \operatorname{dn} \varphi-\varepsilon C(3 I) \operatorname{sn}^{2} \varphi \operatorname{dn}^{2} \varphi
\end{array}\right.
$$

where $\operatorname{cn} \varphi, \operatorname{sn} \varphi, \operatorname{dn} \varphi$ are the cosine-amplitude, sine-amplitude, delta-amplitude functions, respectively, with elliptic modulus $k=1 / \sqrt{2}[25]$.

Let $K(k)$ be the complete elliptic integral of the first type. For $\varepsilon=0$ the periodic solution to 2.1) with frequency $\omega=p / q$ is of the form $x(t)=\alpha \mathrm{cn}\left(\alpha\left(t+t_{0}\right)\right)$, with $2 \pi \alpha=4 \omega K(1 / 2)$ and $t_{0}$ suitably fixed [5]. In terms of action-angle variable this gives $I=I_{0}:=\alpha^{3} / 3$ and $\varphi=\varphi_{0}(t):=\alpha\left(t+t_{0}\right)$.

Linearisation of (A.5) around the periodic solution leads to the system

$$
\left(\begin{array}{c}
\dot{\delta \varphi} \\
\dot{\delta I}
\end{array}\right)=L(t)\left(\begin{array}{c}
\delta \varphi \\
\delta I
\end{array}\right), \quad L(t)=L_{0}+\varepsilon L_{1}(t)+O\left(\varepsilon^{2}\right)
$$

where

$$
L_{0}(t)=\left(\begin{array}{cc}
0 & \alpha^{-2} \\
0 & 0
\end{array}\right), \quad L_{1}(t)=\left(\begin{array}{cc}
L_{11}(t) & L_{12}(t) \\
L_{21}(t) & L_{22}(t)
\end{array}\right)
$$

with

$$
\begin{aligned}
& L_{11}(t)=f(t) \dot{a}(t)-C \alpha^{-1} \dot{b}(t), \quad L_{12}(t)=-2 \alpha^{-5} I_{1}(t)+\alpha^{-2} f(t) a(t), \\
& L_{21}(t)=\alpha^{3} f(t) \dot{c}(t)-C \alpha^{2} \dot{d}(t), \quad L_{22}(t)=4 \alpha f(t) c(t)-3 C d(t),
\end{aligned}
$$

where we have defined

$$
\begin{aligned}
& a(t):=\operatorname{cn}^{4} \varphi_{0}(t), \quad b(t):=\operatorname{cn} \varphi_{0}(t) \operatorname{sn} \varphi_{0}(t) \operatorname{dn} \varphi_{0}(t), \\
& c(t):=\operatorname{cn}^{3} \varphi_{0}(t) \operatorname{sn} \varphi_{0}(t) \operatorname{dn} \varphi_{0}(t), \quad d(t):=\operatorname{sn}^{2} \varphi_{0}(t) \operatorname{cn}^{2} \varphi_{0}(t)
\end{aligned}
$$

and denoted by $\left(\varphi_{1}(t), I_{1}(t)\right)$ the first order of the periodic solution. 
Let us denote by $W(t)=W_{0}(t)+\varepsilon W_{1}(t)+O\left(\varepsilon^{2}\right)$ the Wronskian matrix, that is the matrix whose columns are two independent solutions of the linearised system A.2), so that $\dot{W}(t)=L(t) W(t)$, with $W(0)=\mathbb{1}$. Then one has

$$
W_{0}(t)=\exp t L_{0}=\left(\begin{array}{cc}
1 & \alpha^{-2} t \\
0 & 1
\end{array}\right)
$$

while $W_{1}(t)$ is obtained by solving the system $\dot{W}_{1}=L_{0} W_{1}+L_{1} W_{0}(t)$, i.e.

$$
W_{1}(t)=W_{0}(t)\left[W_{1}(0)+\int_{0}^{t} \mathrm{~d} \tau\left(W_{0}(\tau)\right)^{-1} L_{1}(\tau) W_{0}(\tau)\right]
$$

where one has to take $W_{1}(0)=0$ in order to have $W(0)=\mathbb{1}$.

A trivial computation shows that in A.10 one has

$$
W_{0}(t)^{-1} L_{1}(t) W_{0}(t)=\left(\begin{array}{cc}
L_{11}(t)-\alpha^{-2} t L_{21}(t) & L_{12}(t)+\alpha^{-2} t\left(L_{11}(t)-L_{22}(t)-\alpha^{-2} L_{21}(t)\right) \\
L_{21}(t) & L_{22}(t)+\alpha^{-2} t L_{21}(t)
\end{array}\right) .
$$

Let $T=2 \pi q$ be the period of the periodic solution. The Floquet multipliers around the periodic solution are the eigenvalues of the Wronskian matrix, computed at time $T$. Denote by $x^{k}(t)$ the $k$ th primitive of any function $x(t)$ with $x^{k}(0)=0$ (so that $\dot{x}^{k}(t)=x^{k-1}(t)$, with $x^{0}(t)=x(t)$ ). Then, by using that

$$
\begin{aligned}
& \int_{0}^{T} \mathrm{~d} t x(t)=x^{1}(T), \quad \int_{0}^{T} \mathrm{~d} t t x(t)=T x^{1}(T)-x^{2}(T), \\
& \int_{0}^{T} \mathrm{~d} t t^{2} x(t)=T^{2} x^{1}(T)-2 T x^{2}(T)+2 x^{3}(T)
\end{aligned}
$$

we obtain that

$$
W_{1}(T)=\left(\begin{array}{cc}
L_{11}^{1}(T)+\alpha^{-2} L_{21}^{2}(T) & L_{12}^{1}(T)+\alpha^{-2}\left(T L_{11}^{1}(T)-L_{11}^{2}+L_{22}^{2}+\alpha^{-2}\left(T L_{21}^{2}-2 L_{21}^{3}(T)\right)\right) \\
L_{21}^{1}(T) & L_{22}^{1}(T)+\alpha^{-2}\left(T L_{21}^{1}(T)-L_{21}^{2}(T)\right)
\end{array}\right) .
$$

For $\varepsilon=0$, the corresponding Floquet multipliers are equal to 1 . To first order they are the roots $\lambda_{ \pm}$ of the equation $\lambda^{2}-2 b_{0} \lambda+c_{0}=0$, with

$$
b_{0}:=1+\frac{\varepsilon}{2}\left(L_{11}^{1}(T)+L_{22}^{1}(T)+\alpha^{-2} T L_{21}^{1}(T)\right), \quad c_{0}:=1+\varepsilon\left(L_{11}^{1}(T)+L_{22}^{1}(T)\right),
$$

so that

$$
\lambda_{ \pm}=1 \pm \sqrt{\varepsilon \alpha^{-2} T L_{21}^{1}(T)}+\frac{\varepsilon}{2}\left[L_{11}^{1}(T)+L_{22}^{1}(T)+\alpha^{-2} T L_{21}^{1}(T)\right]+o(\varepsilon) .
$$

One has

$$
L_{11}^{1}(T)+L_{22}^{1}(T)=\int_{0}^{T} \mathrm{~d} t f(t)(\dot{a}(t)+4 \alpha c(t))-C \int_{0}^{T} \mathrm{~d} t\left(\alpha^{-1} \dot{b}(t)+3 d(t)\right)
$$

One immediately realises that the first integral vanishes and hence

$$
L_{11}^{1}(T)+L_{22}^{1}(T)=-3 C T \mu, \quad \mu:=\frac{1}{T} \int_{0}^{T} \mathrm{~d} t d(t)>0,
$$


while, for the stable periodic solution, $t_{0}$ is such that $L_{21}^{1}(T)<0$. Therefore the Floquet multipliers are of the form

$$
\lambda_{ \pm}=1 \pm \mathrm{i} \lambda_{0} \sqrt{T \varepsilon}-\left(\lambda_{0}^{2}+3 C \mu\right) T \varepsilon+o(\varepsilon),
$$

with $\lambda_{0}>0$. The corresponding Lyapunov exponents, defined as $T^{-1} \operatorname{Re} \log \lambda_{ \pm}$, are given by $-3 \mu C \varepsilon=-3 \mu \gamma$. This shows that for primary resonances of the system (2.1), at least for initial conditions close enough to the attractors, convergence to the attractors has rate $1 / \gamma$. In principle the analysis can be extended to any resonance, by writing $\gamma=C \varepsilon^{m}$ and going up to order $m$, for suitable $m$ depending on the resonance $(m=n(\omega)$ for the resonance with frequency $\omega$; see Section 2): the contributions to the Lyapunov exponents due to the Hamiltonian components of the vector fields cancel out and the leading part of the remaining part turns out proportional to $-\gamma$.

In the case of the linearly increasing friction 3.2 one expects that the Lyapunov exponent be still proportional to $-\gamma$. If the friction increases very slowly, one may reason as it were nearly constant over long time intervals, that is time intervals covering several periods, by approximating $\gamma(t)$ with a piecewise constant function. For each of such interval $\gamma$ can be considered as constant and one can reason as above. When passing from an interval to another, the value of the initial phase $t_{0}$ of the attractor slightly changes. The Lyapunov exponent is then expected to behave proportionally to

$$
-\lim _{\tau \rightarrow \infty} \frac{1}{\tau} \int_{0}^{\tau} \mathrm{d} t \gamma(t), \quad \int_{0}^{\tau} \mathrm{d} t \gamma(t)=\int_{0}^{T_{0}} \mathrm{~d} t \gamma(t)+\gamma_{0}\left(\tau-T_{0}\right)=\frac{\Delta}{2}+\left(\gamma_{0} \tau-\Delta\right),
$$

where we have used that $T_{0}=\Delta / \gamma_{0}$. Therefore again the rate of exponential convergence to the attractor is proportional to $1 / \gamma_{0}$ and after the time $T_{0}$ the distance to the attractor has already decreased by a factor $\exp \left(-c_{0} \Delta\right)=\exp \left(-c_{0} \gamma_{0} T_{0}\right)$, for some positive constant $c_{0}$, and hence like in the case of constant friction, possibly with a different constant $c_{0}$.

\section{B Parameters $\varepsilon$ and $\gamma$ for the spin-orbit model}

The spin-orbit model has been extensively used in the literature to study the behaviour of regular satellites [23, 36] - it does not apply to irregular satellites, which are very distant from the planet and follow an inclined, highly eccentric and often retrograde orbit. The equations of motion are given by

$$
\ddot{\theta}+\varepsilon G(\theta, t)=0,
$$

with $G$ as in 4.2 . Here time has been rescaled $t \rightarrow \omega_{T} t$, where $\omega_{T}$ is the mean angular velocity of the satellite along its elliptic orbit (cf. Table B.1), so that the orbital period ('year') of the satellite becomes 1 . Then the $1: 1$ resonance is $\dot{\theta} \approx 1$.

In a system satellite-primary there can be several types of friction: for instance the friction between the satellite layers of different composition, say one liquid and one solid (core-mantle friction), or the friction due to the tides (tidal friction). One can expect that such phenomena produce a friction to be minimised in a 1:1 resonance. There could be also other sources of friction which we do not consider, especially those which could modify the revolution motion of the satellite, because we are implicitly using that it occurs on a fixed orbit. The dissipation term due to tidal torques is of the form [34, 23, 41, 15]

$$
-\gamma(\Omega(e) \dot{\theta}-N(e))
$$


Table B.1: Values of $\omega_{T}$ (angular velocity), $M$ (satellite mass), $M_{0}$ (primary mass), $R$ (satellite radius) and $\rho$ (mean distance between satellite and primary) for the systems considered in Section 4. CGS units are used.

\begin{tabular}{|l|l|l|l|l|l|l|l|}
\hline & Primary & Satellite & $\omega_{T}$ & $M$ & $M_{0}$ & $R$ & $\rho$ \\
\hline \hline E-M & Earth & Moon & $2.66 \times 10^{-6}$ & $7.35 \times 10^{25}$ & $5.97 \times 10^{27}$ & $1.74 \times 10^{8}$ & $3.84 \times 10^{10}$ \\
\hline S-M & Sun & Mercury & $8.27 \times 10^{-7}$ & $3.30 \times 10^{26}$ & $1.99 \times 10^{33}$ & $2.44 \times 10^{8}$ & $5.79 \times 10^{12}$ \\
\hline J-G & Jupiter & Ganymede & $1.02 \times 10^{-5}$ & $1.48 \times 10^{26}$ & $1.90 \times 10^{30}$ & $2.63 \times 10^{8}$ & $1.07 \times 10^{11}$ \\
\hline J-I & Jupiter & Io & $4.11 \times 10^{-5}$ & $8.93 \times 10^{25}$ & $1.90 \times 10^{30}$ & $1.82 \times 10^{8}$ & $4.22 \times 10^{10}$ \\
\hline S-E & Saturn & Enceladus & $5.31 \times 10^{-5}$ & $1.08 \times 10^{23}$ & $5.68 \times 10^{29}$ & $2.52 \times 10^{7}$ & $2.38 \times 10^{10}$ \\
\hline S-D & Saturn & Dione & $2.66 \times 10^{-5}$ & $1.09 \times 10^{24}$ & $5.68 \times 10^{29}$ & $5.62 \times 10^{7}$ & $3.77 \times 10^{10}$ \\
\hline
\end{tabular}

where $\Omega(e)$ and $N(e)$ are two constants depending on $e$. Since both $\Omega(e)=1+O\left(e^{2}\right)$ and $N(e)=$ $1+O\left(e^{2}\right)$, for small values of $e$ we can approximate (B.1) as in (4.1).

A comparison with the literature [23, 15] gives

$$
\varepsilon=\frac{3}{2} \frac{I_{y}-I_{x}}{I_{z}} \approx \frac{3 h}{2 R}, \quad \gamma=\frac{3 k_{2}}{\xi Q}\left(\frac{R}{\rho}\right)^{3}\left(\frac{M_{0}}{M}\right),
$$

where $I_{x}, I_{y}, I_{z}$ are the moments of inertia of the satellite, $h$ is the maximal equatorial deformation (tide excursion), $R$ and $M$ are the mean radius and the mass of the satellite, $M_{0}$ is the mass of the primary, $\rho$ is the mean distance between satellite and primary and $k_{2}, \xi, Q$ are constants, known respectively as the potential Love number, the structure constant and the quality factor. For instance, in the case of the Moon one has $k_{2} \approx 0.02, \xi \approx 0.4$ and $Q \approx 30\left[28\right.$, 61, which gives $3 k_{2} / \xi Q \approx 0.005$ and hence $\gamma \approx 3.75 \times 10^{-8}$ (approximately $3.15 \times 10^{-6}$ years $^{-1}$ ). In the case of $\mathrm{S}-\mathrm{M}$, the constants are usually (somehow arbitrarily) set equal to the values $k_{2} \approx 0.4, \xi \approx 0.3333$ and $Q \approx 50$ [23, 24, 54, 27, which gives $3 k_{2} / \xi Q \approx 0.072$. The corresponding value of the damping coefficient is $\gamma \approx 3.24 \times 10^{-8}$, a value very close to Mercury's. Expressed in years ${ }^{-1}$ this becomes approximately $8.46 \times 10^{-7}$ (the revolution period of Mercury is $2 \pi / \omega_{T} \approx 7.60 \times 10^{6} \mathrm{~s} \approx 0.24$ year). For lack of astronomical data we set $3 k_{2} / \xi Q=10^{-1}$ for all other primary-satellite systems considered in Section 4 the corresponding values of $\gamma$ as obtained from (B.3) are given in TableB.2. Of course such values only provide a rough guide.

Table B.2: Values of $T$ (orbital period) and $\gamma$ for the systems considered in Section 4 , with $3 k_{2} / \xi Q=0.1$ for the systems with Jupiter and Saturn as primary. In the third column $\gamma$ is computed by using $T$ as time unit, whereas the fourth column gives the value of the damping coefficient expressed in years ${ }^{-1}$.

\begin{tabular}{|l|l|l|l|l|l|l|}
\hline \hline & Primary & Satellite & $T$ & $T$ (years) & $\gamma$ & $\gamma\left(\right.$ years $\left.^{-1}\right)$ \\
\hline \hline E-M & Earth & Moon & $2.36 \times 10^{6}$ & $7.48 \times 10^{-2}$ & $3.75 \times 10^{-8}$ & $3.15 \times 10^{-6}$ \\
\hline S-M & Sun & Mercury & $7.60 \times 10^{6}$ & $2.41 \times 10^{-1}$ & $3.24 \times 10^{-8}$ & $8.46 \times 10^{-7}$ \\
\hline J-G & Jupiter & Ganymede & $6.18 \times 10^{5}$ & $1.96 \times 10^{-2}$ & $1.91 \times 10^{-5}$ & $2.48 \times 10^{-2}$ \\
\hline J-I & Jupiter & Io & $1.53 \times 10^{5}$ & $4.84 \times 10^{-3}$ & $1.71 \times 10^{-4}$ & $5.51 \times 10^{-2}$ \\
\hline S-E & Saturn & Enceladus & $1.18 \times 10^{5}$ & $3.75 \times 10^{-3}$ & $6.26 \times 10^{-4}$ & 1.05 \\
\hline S-D & Saturn & Dione & $2.36 \times 10^{5}$ & $7.49 \times 10^{-3}$ & $1.71 \times 10^{-4}$ & $1.44 \times 10^{-1}$ \\
\hline
\end{tabular}


To obtain the value of $\varepsilon$ one can use the formula

$$
h=\frac{3}{2} h_{2} R\left(\frac{R}{\rho}\right)^{3}\left(\frac{M_{0}}{M}\right),
$$

for the equatorial deformation; see Appendix C. Here $h_{2}$ is the tidal Love number $\left(h_{2} \approx 2 k_{2}\right.$ [61]), while the other constants are as defined after (B.3). If we are interested only in orders of magnitude, we can express the equatorial deformation according to (B.4) with $h_{2}=1$ for the systems with Jupiter or Saturn as primary. Then, by inserting the values of $R, \rho, M_{0}, M$ listed in Table B.1 into (B.4) and using (B.3) to compute $\varepsilon$, we obtain the values in Table 4.1. A comparison between (B.3) and (B.4) gives $\gamma=C \varepsilon$, with $C \approx 0.05$ (taking the values of the constants $k_{2}, \xi, Q$ and $h_{2}$ in the literature gives $C \approx 0.04$ for $\mathrm{E}-\mathrm{M}$ and $C \approx 0.055$ for $\mathrm{S}-\mathrm{M})$.

Note that the values so obtained for $\varepsilon$ are lower than those usually assumed in the literature: compare for instance the values $\varepsilon=2.3 \times 10^{-4}$ for E-M [58] and $\varepsilon=1.5 \times 10^{-4}$ for S-M [3, 15, 16, 13, 27. with the corresponding values $\varepsilon=6.75 \times 10^{-7}$ and $\varepsilon=8.11 \times 10^{-7}$ in Table 4.1. However, as discussed in Section 5, if one does not insist at looking only at the present structure of the satellite, then all its evolution plays a relevant role. So, one has to take into account that in the past, when the satellite was more fluid, because of the lower value of viscosity, not only the friction was smaller, but also the deformation was bigger and hence the coupling $\varepsilon$ was larger; see also the comments in Section 6 .

\section{The equatorial deformation}

Consider a homogeneous celestial body $S$ of mean radius $R$ coated by an ocean of depth $h>0$, not too small. Let $P$ be the centre of attraction. Denote by $M$ and $M_{0}$ the respective masses and assume that the motion of the two celestial bodies about their centre of mass be circular uniform. Let $\rho$ be the distance between the two celestial bodies $S$ and $P$, with $\rho \gg R \gg h$. Assume, for simplicity, that $S$ rotates about an axis orthogonal to the plane of the orbit and that the ocean density is negligible with respect to the core assumed to be rigid. The discussion below is essentially taken from [36].

The distance $\rho_{C}$ of the centre of mass $C$ from the centre of $S$ is such that $\rho_{C}\left(M_{0}+M\right)=M_{0} \rho$. Moreover, if $\omega_{T}$ denotes the angular velocity of revolution of the two celestial bodies and $\kappa$ is the universal gravitational constant, one has $\omega_{T}^{2} \rho^{3}=\kappa\left(M+M_{0}\right)$ by Kepler's third law. Let $n$ be a unit vector out of the surface of $S$ and note that, imagining the observer standing on the frame of reference rotating around $C$ with angular velocity $\omega$, so that the axis from $P$ to $S$ has a fixed unit vector $i$, the potential (gravitational plus centrifugal) energy in the point along the direction $n$ at distance $r$ from the centre of $S$ has density $V=V_{S}+V_{P}+V_{\text {cf }}$, where

$$
V_{S}=-\kappa \frac{M}{r}, \quad V_{P}=-\kappa \frac{M_{0}}{\left(\rho^{2}+r^{2}-2 \rho r \cos \psi\right)^{1 / 2}}, \quad V_{\mathrm{cf}}=\frac{1}{2} \omega^{2}\left(\rho_{C}^{2}+r^{2}-2 \rho_{C} r \cos \psi\right),
$$

if $\cos \psi:=i \cdot n$. Expanding $V$ in powers of $r / \rho$ one finds

$$
V_{P}+V_{\mathrm{cf}}=-\kappa \frac{M}{\rho}\left(\frac{r}{\rho}\right)^{2}\left(\frac{3}{2} \frac{M_{0}}{M} \cos ^{2} \psi+\frac{1}{2}\right)+\text { const. }
$$

because the linear terms cancel out in virtue of Kepler's third law. Therefore the equation of the equipotential surface is

$$
\frac{\rho}{r}+\left(\frac{r}{\rho}\right)^{2}\left(\frac{3}{2} \frac{M_{0}}{M} c^{2}+\frac{1}{2}\right)=\text { const. }
$$


If one writes $r=R(1+\delta(\psi))$, with $\delta(\psi)=\delta_{0}+\delta P_{2}(\cos \psi)$, where $P_{2}(z)=\left(3 z^{2}-1\right) / 2$ is the second Legendre polynomial [25] and $\delta_{0}$ is such that the volume of the body $P$ is the same as the volume of a sphere of radius $R$, then $(\mathrm{B} .3)$ gives

$$
\delta(\psi)=\delta P_{2}(\cos \psi), \quad \delta_{0}=0, \quad \delta=\left(\frac{R}{\rho}\right)^{3} \frac{M_{0}}{M} .
$$

If the core of the satellite is rigid but the ocean density $\sigma_{\mathrm{o}}$ is not negligible, e.g. it is equal to the core density $\sigma_{\mathrm{c}}$, then one has to take into account that the tide will modify the potential $V_{S}$ at the site of coordinates $r, \psi$. We make the Ansatz that the equipotential surface is still described as $r=R\left(1+\delta P_{2}(\cos \psi)\right)$, possibly for a different constant $\delta$. Then the density $V_{S}$ will be

$$
-\frac{3 \kappa M}{4 \pi R^{3}} \int_{0}^{\pi} \sin \alpha \mathrm{d} \alpha \int_{0}^{2 \pi} \mathrm{d} \varphi \int_{0}^{R\left(1+\delta P_{2}(\cos \alpha)\right)} \frac{\rho^{2} \mathrm{~d} \rho}{\left(r^{2}+\rho^{2}-2 r \rho(\cos \psi \cos \alpha+\sin \psi \sin \alpha \cos \varphi)\right)^{1 / 2}} .
$$

By expanding the integrand into Legendre polynomials and using the orthogonality properties of the polynomials one finds (see [36], §4.3 for details)

$$
V_{S}= \begin{cases}-\kappa M\left(\frac{3 R^{2}-r^{2}}{2 R^{3}}+\frac{3}{5} \frac{r^{2}}{R^{3}} \delta P_{2}(\cos \psi)\right), & r<R, \\ -\kappa M\left(\frac{1}{r}+\frac{3}{5} \frac{R^{2}}{r^{3}} \delta P_{2}(\cos \psi)\right), & r>R .\end{cases}
$$

By expanding (C.6) in powers of $r / \rho$ and summing the leading orders to (C.2), one finds that the equation of the equipotential surface becomes

$$
\frac{\kappa M}{\rho}\left(\frac{\rho}{R}\left(1-\frac{2}{5} \delta P_{2}(\cos \psi)\right)+\left(\frac{r}{\rho}\right)^{2} \frac{M_{0}}{M} P_{2}(\cos \psi)\right)=\text { const. }
$$

up to higher order corrections in $R / \rho$. Hence if we look for the constant potential surface we find

$$
\delta(\psi)=\delta P_{2}(\cos \psi), \quad \delta=\frac{5}{2}\left(\frac{R}{\rho}\right)^{3} \frac{M_{0}}{M},
$$

which replaces the previous (C.4). The tidal deformation at the surface of the ocean, using the notations common in celestial mechanics, can be written as $h_{2} \zeta P_{2}(\cos \psi)$, so that the maximal tidal excursion is

$$
h=\frac{3}{2} h_{2} \zeta, \quad \zeta=R\left(\frac{R}{\rho}\right)^{3} \frac{M_{0}}{M}, \quad h_{2}=\frac{5}{2} .
$$

The number $h_{2}$ is called the tidal Love number. More generally $h_{2}$ depends on the detailed structure of the satellite, so far supposed to have uniform density $\sigma_{\mathrm{o}}=\sigma_{\mathrm{c}}$. If on the contrary $\sigma_{\mathrm{o}} \neq \sigma_{\mathrm{c}}$, then, denoting by $r_{\mathrm{c}}$ the shape of the core boundary (while $r$ is the shape of the external ocean surface), one can make once more the Ansatz that the deformations be such that $r=R\left(1+\delta P_{2}(\cos \psi)\right)$ and $r_{\mathrm{c}}=R_{c}\left(1+\delta^{\prime} P_{2}(\cos \psi)\right)$, where $R_{\mathrm{c}}$ is the mean radius of the core and $\delta, \delta^{\prime}$ are two constants to be determined by imposing that the ocean surface is equipotential and balancing the forces acting on the core boundary. The latter can be performed by considering the pressures acting on the core 
boundary due to the elastic forces within the core and the loaded terms caused by the ocean and core tide. This leads to two relations involving $\delta, \delta^{\prime}$ (see [36], §4.4)

$$
\begin{aligned}
\frac{\zeta_{\mathrm{c}}}{R_{\mathrm{c}}} & =\left[\frac{2}{5} \frac{\sigma_{\mathrm{o}}}{\sigma_{\mathrm{c}}}+\left(\frac{R_{\mathrm{c}}}{R}\right)^{3}\left(1-\frac{\sigma_{\mathrm{o}}}{\sigma_{\mathrm{c}}}\right)\right] \delta^{\prime}-\frac{3}{5}\left(\frac{R_{\mathrm{c}}}{R}\right)^{5}\left(1-\frac{\sigma_{\mathrm{o}}}{\sigma_{\mathrm{c}}}\right) \delta, \\
\delta & =\frac{1}{\tilde{\mu}}\left(1-\frac{\sigma_{\mathrm{o}}}{\sigma_{\mathrm{c}}}\right)\left[\frac{5}{2} \frac{\zeta_{\mathrm{c}}}{R_{\mathrm{c}}}-\delta+\frac{3}{2} \frac{\sigma_{\mathrm{o}}}{\sigma_{\mathrm{c}}}\left(\delta^{\prime}-\delta\right)\right],
\end{aligned}
$$

where $\zeta_{\mathrm{c}}=\left(M / M_{\mathrm{c}}\right) \zeta$ (with $M_{\mathrm{c}}$ being the mass of the core) and the effective rigidity $\tilde{\mu}$ is a dimensionless quantity proportional to the rigidity of the core. For instance, if $\sigma_{\mathrm{o}} \ll \sigma_{\mathrm{c}}$, we can approximate

$$
R_{\mathrm{c}} \delta^{\prime} \approx \frac{5}{2} \frac{\zeta_{\mathrm{c}}}{1+\tilde{\mu}}, \quad R \delta \approx \zeta_{\mathrm{c}}\left[\left(\frac{R}{R_{\mathrm{c}}}\right)^{4}+\frac{3}{2}\left(\frac{R_{\mathrm{c}}}{R}\right) \frac{1}{1+\tilde{\mu}}\right] .
$$

In particular in the limit of high rigidity $(\mu \gg 1)$ then $R_{\mathrm{c}} \delta^{\prime} \approx 0$ and $R \delta \approx\left(R / R_{\mathrm{c}}\right)^{4} \zeta_{\mathrm{c}}=\zeta$ (in agreement with (B.4) , so that the core deformation becomes very small, that is the core is essentially undeformed.

\section{Threshold values for the spin-orbit model}

We reason as done for the driven cubic oscillator in [5]. We consider (4.1) with $\gamma=\varepsilon C$ and write it as the first order differential equation

$$
\left\{\begin{array}{l}
\dot{\theta}=y \\
\dot{y}=-\varepsilon G(\theta, t)-\varepsilon C(y-1)
\end{array}\right.
$$

Then we look for a solution $z(t)=(\theta(t), y(t))$ in the form of a power series in $\varepsilon$, that is $z(t)=z^{(0)}(t)+$ $\varepsilon z^{(1)}(t)+\varepsilon^{2} z^{(2)}(t)+\ldots$, where $z^{(0)}(t)=\left(\theta_{0}+\omega t, \omega\right)$, with $\omega=p / q$, and $z^{(k)}(t)=\left(\theta^{(k)}(t), y^{(k)}(t)\right)$ to be determined by imposing that $z(t)$ be periodic in $t$ with period $2 \pi q$.

A first order analysis gives

$$
\left\{\begin{array}{l}
\dot{\theta}^{(1)}=y^{(1)} \\
\dot{y}^{(1)}=-G\left(\theta_{0}+\omega t, t\right)-C(\omega-1) .
\end{array}\right.
$$

By introducing the Wronskian matrix

$$
W(t)=\left(\begin{array}{cc}
1 & \omega t \\
0 & \omega
\end{array}\right)
$$

we can write $z^{(1)}(t)$ as

$$
\left(\begin{array}{l}
\theta^{(1)}(t) \\
y^{(1)}(t)
\end{array}\right)=W(t)\left(\begin{array}{c}
\bar{\theta}^{(1)} \\
\bar{y}^{(1)}
\end{array}\right)+W(t) \int_{0}^{t} \mathrm{~d} \tau W^{-1}(\tau)\left(\begin{array}{c}
0 \\
-G\left(\theta_{0}+\omega \tau, \tau\right)-C(\omega-1)
\end{array}\right)
$$

with $\left(\bar{\theta}^{(1)}, \bar{y}^{(1)}\right)$ to be fixed. Then we obtain

$$
\theta^{(1)}(t)=\bar{\theta}^{(1)}+\bar{y}^{(1)} \omega t-\int_{0}^{t} \mathrm{~d} \tau \int_{0}^{\tau} \mathrm{d} \tau^{\prime}\left[G\left(\theta_{0}+\omega \tau^{\prime}, \tau^{\prime}\right)+C(\omega-1)\right]
$$


whereas $y^{(1)}(t)=\dot{\theta}^{(1)}(t)$. For (D.4) to be periodic we have to require first of all that

$$
M\left(\theta_{0}\right):=\frac{1}{2 \pi q} \int_{0}^{2 \pi q} \mathrm{~d} t\left[G\left(\theta_{0}+\omega t, t\right)+C(\omega-1)\right]=0,
$$

then fix $\bar{y}^{(1)}$ in such a way that

$$
\bar{y}^{(1)} \omega=\frac{1}{2 \pi q} \int_{0}^{2 \pi q} \mathrm{~d} t \int_{0}^{t} \mathrm{~d} \tau\left[G\left(\theta_{0}+\omega \tau, \tau\right)+C(\omega-1)\right]
$$

while $\bar{\theta}^{(1)}$ will be fixed to second order by requiring that also $\theta^{(2)}(t)$ be periodic.

Using that $G(\theta, t)=\partial_{\theta} g(\theta, t)$, with $g(\theta, t)$ given by (4.3), and inserting (D.6) into (D.5) leads to

$$
\frac{1}{2 \pi q} \sum_{k \in \mathbb{Z}} 2 a_{k} \int_{0}^{2 \pi q} \mathrm{~d} t \sin \left(2 \theta_{0}+2 \omega t-k t\right)=C(\omega-1)
$$

and hence

$$
2 a_{k(p)} \sin 2 \theta_{0}=C\left(\frac{p}{q}-1\right), \quad k(p)=\frac{2 p}{q} .
$$

Since $a_{k} \neq 0$ only for $k=-3, \ldots, 7, k \neq 0$, as 4.2 implies, $\omega=p / q$ is either integer (and $\omega \in\{-1,1,2,3\}$ ) or half-integer (and $\omega \in\{-3 / 2,-1 / 2,1 / 2,3 / 2,5 / 2,7 / 2\}$ ). If we confine ourselves to positive $\omega$, we see that D.9 fixes $\theta_{0}$ provided

$$
|C|<C_{0}(p / q):=\frac{2 a_{k(p)} q}{|p-q|}
$$

In particular D.10 is always satisfied for $\omega=1$, so that the 1:1 resonance always exists, while for the other values of $\omega$ we obtain 4.4 .

\section{E Time evolution of friction for the spin-orbit model}

The mechanism of capture into resonance has been studied by several authors starting with the theory of capture into the 3:2 resonance of Mercury [23, 12, 37]. Usually the friction is considered either periodic or just not depending on time. Here we regard the friction as not periodic in time and given by (2.2), that is starting from a initial very small value, then slowly increasing in order of magnitude, until the satellite has completely solidified: such a situation seems possible in the formation of a satellite or planet. At the beginning, the satellite can be considered in a fluid state; however the dissipation due to tidal effects becomes more and more sensible due to the cooling and the resulting increase of viscosity and, eventually, it settles at the final present value: the time over which the entire process takes place is called the solidification time and will be denoted by $T_{S}$.

We stress that, in the model we are considering, we assume that the satellite has first stabilised in its orbit around the primary and then modifies its spinning velocity. Of course the exact evolution of the satellite dynamics is still debated and no theory is universally agreed upon. In what follows, we shall ignore the model-dependent details of such an evolution. So, for instance, if we accept that in some stage of the history of Mercury large quantities of its mantle material have been removed 
[60, 8], for the purposes of our argument it is not important whether this occurred before Mercury attained its final orbital motion or after that event.

Suppose that friction is essentially due to tidal effects on an originally entirely fluid fast rotating satellite (that is with rotation frequency $\dot{\theta}$ at least a few times larger than the present-day orbital frequency $\omega_{T}$ ) evolving toward a solid body. Assuming that the dynamics is described by (4.1) since the beginning could appear contradictory with the fact that originally the satellite was essentially fluid, since (4.1) deals with a rigid body with given moments of inertia. On the other hand, as we shall argue, friction becomes really effective only when the viscosity has attained high values and, when this occurs, the shape of the satellite can be considered close to its final state. One could also imagine to study the deformations of the satellite during its evolution, but of course this would make the analysis much more complicated; see for instance [4], where asymptotic stability of the 1:1 resonance is obtained for a deformable body with high rigidity (see also [50]). In other words, using the spin-orbit model is justified except possibly for the very early stage of the satellite evolution (which are not interested in).

In the early history of the solar system one can assume that the viscosity is rather low, not too different from that of the water, which equals $10^{-2}$ poises (CGS units). The solidification process is very complicated, and although it has been extensively studied in the literature, especially in the case of the Earth and the Moon (for the obvious reason that it is much easier to compare the results obtained by theoretical methods and numerical simulations with the experimental data), still there are many unsolved issues. See for instance [51] for a review of recent results on the Moon. Usually one assumes that originally all satellites and planets were completely or almost completely molten, according to the so-called magma ocean hypothesis; in the case of the Moon see [57] and especially [51] and the references quoted therein (see also [55] in the case of Earth). Most of the celestial body crystallised, from the bottom to the top, following a sequence determined by chemical composition and pressure [52, 29, 18] leaving only a layer of very fluid magma close to the surface of the satellite. The outer liquid part eventually disappeared, through a further solidification process from the coremantle boundary to the surface, and it is irrelevant in any case to the damping, because of its very low viscosity.

Timing of formation of satellites and for the cooling of the magma is mostly deduced from the study of rock samples: in the case of the Moon of course this is much easier [51, 10, 17, 38, while the results are far from being conclusive in the case of Mercury (however see, for instance, [53, 11]). In any case, it is not unlikely that the solidification time of Mercury is of the same order of magnitude of that of the Moon; we can also mention that the theory has been proposed that the two celestial bodies may have had similar origin [59, 60]. There is strong evidence that the solidification time $T_{S}$ for the Moon is of order of $10^{8}$ years [10, 38]. Thus, for the reasons given above, we take this as the order of magnitude of the solidification time of all the satellites considered in Section 4 .

For most of the solidification process the ocean magma is maintained above its solidus [1, 18]. So it is reasonable that the viscosity has increased as an effect of the cooling of the satellite: eventually most of the satellite is almost solidified and its viscosity is very large, say of the order of that of the mantle, which is almost solid (hence about $10^{24}$ poises, much higher than the viscosity of the terrestrial magma which ranges from $10^{4}$ and $10^{12}$ poises [42, 30]). Of course the viscosity strongly depends on temperature, which in turn decreases in time during the process of cooling of the satellite. One could look at the literature for profiles of temperatures versus time [55, 21] or for the dependence of viscosity on temperature in fluids [49]. A detailed discussion on viscosity evolution during the 
solidification of the satellite stands as a very hard problem, and in fact such an issue is not widely studied [45, 18, 51]; see however [46], where the despinning of Saturn's satellite Iapetus is studied and an Arrhenius law is assumed to describe the temperature dependence of the viscosity, and [47, where the despinning of Mercury is related to the thermal evolution of the planet.

As far as the satellite can be considered essentially fluid, the damping coefficient $\gamma$ has to be formed with the following physical quantities: the viscosity $\eta$ of the magmatic fluid constituting the satellite, the mass $M$ of the satellite, the equatorial deformation $2 h$ due to the tides and the angular velocity $\omega_{T}$. Starting from the rescaled equation (4.1), one expects $\gamma \propto \eta R^{2} h / I_{z}$, with $I_{z} \propto M R^{2}$; then, by rescaling time $t \rightarrow t / \omega_{T}$, an appropriate choice for $\gamma$ turns out to be $\gamma \sim \eta h / M \omega_{T}$. The evolution of magma from the initial melt passes through the formation of cumulate rocks and fractional crystallisation, leading to the reduction of the outer liquid layer with low viscosity and the accretion of the internal, highly viscous core; for a more detailed discussion see for instance [51] in the case of the Moon. Therefore, when the solidification process attained a high stage of advancement, a different model for the friction has to be taken. If the satellite is essentially solid, with a thin external fluid layer (ocean), one can neglect the fluid part, because of its very low viscosity, and concentrate on the solid core. One can use the analysis in Appendix B, in the limit case $\sigma_{\mathrm{o}} \ll \sigma_{\mathrm{c}}$ and very high rigidity $\mu$, so that the core deformation in (C.10b is very small. Also in this case, one can express $\gamma$ in terms of the involved physical quantities and, again on the basis of dimensional arguments, set

$\gamma \sim \mu h / M \omega_{T}^{2}$. Therefore, to sum up, friction increases with viscosity up to a certain value. Once such a value is reached, at which the satellite can be considered essentially a solid with very high rigidity.

The despinning time $T_{D}$ represents the time which the satellite needs to be really attracted into a resonance. Hence (see Appendix A $T_{D}=O\left(\gamma^{-1}\right)$, where $\gamma$ is the quantity appearing in (4.1). Of course, we would want that the solidification time be larger or at least comparable with despinning time. This is the case if one can assume $T_{S} \approx 10^{8}$ years and $T_{D} \approx 1 / \gamma$, with $\gamma$ as in Table B.2 (which yields $T_{D} \approx 10^{6}$ years).

\section{F Numerical details}

The numerical results have been found by running a variety of computer programs which implement different algorithms. The main algorithms used were (i) a standard Runge-Kutta integrator with automatic step-size control [44]; (ii) the Bulirsch-Stoer algorithm [44, which extrapolates the stepsize to zero and is suitable for high-accuracy computation; and (iii) a numerical implementation of the Frobenius method. Of these, (ii) is the slowest, but serves to confirm the results obtained from the other methods. Most of the data in this paper came from (i) and (iii), of which (iii) is the fastest. This is because the use of series often enables large time steps to be taken. On the assumption that the solution to the differential equation around a point $t=t_{0}$ can be expressed as a power series in $t$, we obtain a (nonlinear) recursion formula for the coefficients in this series. In principle, as many terms as desired can be computed, and in practice about 25 worked well. For a given initial condition, this series enables us to compute $x$ and $\dot{x}$ for $\left|t-t_{0}\right|<R$, for some $R$ that depends on the desired accuracy, the initial conditions, and $t_{0}$. The size of the step, $t-t_{0}$, is chosen as the one that makes the absolute value of the right-hand side of the differential equation, which should of course be zero, smaller than some tolerance: $10^{-12}$ was used here. Typical step sizes ranged between about 0.29 and 10.0, with 0.7 being chosen about $50 \%$ of the time. Even the smallest step size is many 
times larger than that which would be used in a Runge-Kutta implementation.

The initial conditions are chosen randomly and are uniformly distributed inside the square $\mathcal{Q}$. Since we seek detailed estimates of the relative areas of the basins of attraction, at least up to the first decimal digit whenever possible, we have to take many initial conditions: certainly 1000 initial conditions, as in [15, 13] is not enough. For the data to be reliable to the first decimal place, with a $95 \%$ confidence level, we found that 1000000 initial conditions need to be taken inside the square $\mathcal{Q}$ defined in Section 2, A statistically rigorous justification for this confidence level, given the number of initial conditions, can be found in [56, chapter 9]. Thus, for the relative areas in Table 2.3, we used up to 1000000 initial conditions except for smallest values of $\gamma$ (500 000 initial condition for $\gamma=0.00005$, 150000 initial condition for $\gamma=0.00001$ and 50000 initial conditions for $\gamma=0.000005$ ). Analogously we considered 1000000 initial conditions for $\varepsilon=0.5$ (Table 5.1) and 500000 initial conditions for $\varepsilon=0.01$ (Table 5.2). Also in Section 3 we considered as many initial data as possible: 1000000 initial conditions for larger $\gamma(\gamma=0.015), 500000$ for $\gamma=0.005$ and 250000 for the smallest value $\gamma=0.0005$. As a general rule, the smaller $\gamma$ is, the longer the integration time and hence, by taking $\gamma$ smaller, we also need to diminish the number of initial conditions, in order that the computation time does not become prohibitively long. However, the error on the relative areas becomes larger. That said, the general scenario described in Section 3 seems clear and well supported by the numerics.

As already noted in [48, for $\gamma$ very small, the basins of attraction are spread out over the entire phase space and become very sparse. Thus, if one wants to detect which basin a given initial condition belongs to, very high numerical precision is needed.

Finally, the integration time $T_{\text {int }}$ must be chosen in such a way that all trajectories reach the attractor (within a reasonably fixed accuracy). For instance, one can take $T_{\text {int }}=N / \gamma_{0}$, with $N=20$. Therefore, in order to investigate the dynamics for very small values of the damping coefficient, $T_{\text {int }}$ has to be very large.

The conclusion is that we have to follow the trajectories of a large number of initial conditions, for very long times and with very high accuracy. Of course, this is at odds with obtaining results within a a reasonable time, so that we need to reach a compromise. This has led us to the choice described above.

\section{References}

[1] Y. Abe, Thermal and chemical evolution of the terrestrial magma ocean, Phys. Earth Planet. Int. 100 (1997), 27-39.

[2] E.Yu. Aleshkina, Synchronous spin-orbital resonance locking of large planetary satellites, Solar Syst. Res. 43 (2009), no. 1, 71-78.

[3] J.D. Anderson, G. Colombo, P.B. Esposito, E.L. Lau, G.B. Trager, The mass, gravity field, and ephemeris of Mercury, Icarus 71 (1987), 337-349.

[4] D. Bambusi, E. Haus, Asymptotic stability of spin orbit resonance for the dynamics of a viscoelastic satellite, Preprint, 2010.

[5] M.V. Bartuccelli, A. Berretti, J.H.B. Deane, G. Gentile, S. Gourley, Periodic orbits and scaling laws for a driven damped quartic oscillator, Nonlinear Anal. Real World Appl. 9 (2008), no. 5, 1966-1988. 
[6] M.V. Bartuccelli, J.H.B. Deane, G. Gentile, S. Gourley, Global attraction to the origin in a parametrically driven nonlinear oscillator, Appl. Math. Comput. 153 (2004), no. 1, 111.

[7] M.V. Bartuccelli, G. Gentile, K.V. Georgiou, On the dynamics of a vertically driven damped planar pendulum, R. Soc. Lond. Proc. Ser. A Math. Phys. Eng. Sci. 457 (2001), no. 2016, 3007-3022.

[8] W. Benz, A. Anic, J. Horner, J.A. Whitby, The origin of Mercury, Space Sci. Rev. 132 (2007), 189-202.

[9] G.D. Birkhoff, Dynamical systems, American Mathematical Society Colloquium Publications Vol. IX, Providence, R.I., 1966.

[10] A. Brandon, A younger moon, Nature 450 (2007), 1169-1170.

[11] S.M. Brown, L.T. Elkins-Tanton, Compositions of Mercury's earliest crust from magma ocean models, Earth Planet. Sci. Lett. 286 (2009), 446-455.

[12] T.J. Burns, C.K.R.T. Jones, A mechanism for capture into resonances, Phys. D 69 (1993), no. 1-2, 85-106.

[13] A. Celletti, L. Chierchia, Measures of basins of attraction in spin-orbit dynamics, Celestial Mech. Dynam. Astronom. 101 (2008), no. 1-2, 159-170.

[14] Sh.N. Chow, J.K. Hale, Methods of bifurcation theory, Grundlehren der Mathematischen Wissenschaften Vol. 251, Springer, New York-Berlin, 1982.

[15] A.C.M. Correia, J. Laskar, Mercury's capture into the 3/2 spin-orbit resonance as a result of its chaotic dynamics Nature 429 (2004), 848-850.

[16] A.C.M. Correia, J. Laskar, Mercury's capture into the 3/2 spin-orbit resonance including the effect of core-mantle friction, Icarus 201 (2009), 1-11.

[17] V. Debaille, A. D. Brandon, Q. Z. Yin, B. Jacobsen, Coupled ${ }^{142} N d-{ }^{143} N d$ evidence for a protracted magma ocean in Mars, Nature 450 (2007), 525-528.

[18] L.T. Elkins-Tanton, Linked magma ocean solidification and atmospheric growth for Earth and Mars, Earth Planet. Sci. Lett. 271 (2008), 181-191.

[19] U. Feudel,C. Grebogi, Why are chaotic attractors rare in multistable systems, Phys. Rev. E 91 (2003), 134102, 4 pp.

[20] U. Feudel,C. Grebogi, B.R. Hunt, J.A. Yorke, Map with more than 100 coexisting low-period periodic attractors, Phys. Rev E 54 (1996), 71-81.

[21] P.E. Fricker, R.T. Reynolds, A.L. Summers, Possible thermal history of the Moon, in The Moon, Eds. H.C. Urey and S.K. Runcorn, Reidel, Dordrecht, pp. 384-391, 1972.

[22] G. Gentile, M.V. Bartuccelli, J.H.B. Deane, Bifurcation curves of subharmonic solutions and Melnikov theory under degeneracies, Rev. Math. Phys. 19 (2007), no. 3, 307-348.

[23] P. Goldreich, S. Peale, Spin-orbit coupling in the solar system, Astronom. J. 71 (1966), no. 6, 425-438.

[24] P. Goldreich, S. Soter, $Q$ in the Solar system, Icarus 5 (1966), 375-389.

[25] I.S. Gradshteyn, I.M. Ryzhik, Table of integrals, series, and products, Sixth edition, Academic Press, Inc., San Diego, 2000. 
[26] J. Guckenheimer, Ph. Holmes, Nonlinear oscillations, dynamical systems, and bifurcations of vector fields, Applied Mathematical Sciences Vol. 42, Springer, New York, 1990.

[27] T. Van Hoolst, F. Sohl, I. Holin, O. Verhoeven, V. Dehant, T. Spohn, Mercury's interior structure, rotation, and tides, Space Sci. Rev. 132 (2007), 203-227.

[28] A. Khan, K. Mosegaard, J. G. Williams, P. Lognonné, Does the Moon possess a molten core? Probing the deep lunar interior using results from LLR and lunar prospector, J. Geophys. Res. 109 (2004), E09007, $25 \mathrm{pp}$.

[29] Th. Kleine, H. Palme, K. Mezger, A.N. Halliday, Hf-W chronometry of lunar metals and the age and early differentiation of the Moon, Science 310 (2005), no. 5754, 1671-1674.

[30] D.L. Kohlstedt, M.E. Zimmerman, Rheology of partially molten mantle rocks, Ann. Rev. Earth Planet. Sci. 24, (1996), 41-62.

[31] N.N. Krasovsky, Stability of motion. Applications of Lyapunov's second method to differential systems and equations with delay, Stanford University Press, Stanford, Calif. 1963.

[32] J. Laskar, O. Néron de Surgy, On the long term evolution of the spin of the Earth, Astronom. Astrophys. 318 (1997), 975-989.

[33] A.J. Lichtenberg, M.A. Lieberman, Regular and chaotic dynamics, Applied Mathematical Sciences Vol. 38, Springer, New York, 1992.

[34] G.J.F. MacDonald, Tidal friction, Rev. Geophys. 2 (1964), no. 3, 467-541.

[35] W. Magnus, S. Winkler, Hill's equation, Dover Publications, Inc., New York, 1979.

[36] C.D. Murray, S.F. Dermott, Solar system dynamics, Cambridge University Press, Cambridge, 2001.

[37] A.I. Neishtadt, On probabilistic phenomena in perturbed systems, Selecta Math. Sov. 12 (1993), no. 3, 195-210.

[38] A. Nemchin, N. Timms, R. Pidgeon, T. Geisler, S. Reddy, C. Meyer, Timing of crystallisation of the lunar magma ocean constrained by the oldest zircon, Nature Geosci. 2 (2009), 133-136.

[39] J. Palis, A global view of Dynamics and a conjecture on the denseness of finitude of attractors, Astérisque 261 (1995) 335-347.

[40] J. Palis, A global perspective for non-conservative dynamics, Ann. Inst. H. Poincaré Anal. Non Linéaire 22 (2005), no. 4, 485-507.

[41] S.J. Peale, The free precession and libration of Mercury, Icarus 178 (2005), 4-18.

[42] C.C. Plummer, D. McGeary, D.H. Carlson, Physical geology, McGraw-Hill, 2003.

[43] M. Porter, P. Cvitanović, A perturbative analysis of modulated amplitude waves in Bose-Einstein condensates, Chaos 14 (2004), no. 3, 739-755.

[44] W.H. Press, S.A. Teukolsky, W.T. Vetterling and B.P. Flannery, Numerical Recipes in C, Cambridge University Press, Cambridge, UK, 1992.

[45] M.E. Pritchard, D.J. Stevenson, Thermal implications of a lunar origin by giant impact, in Origin of the Earth and Moon, Eds. R.M. Canup and K. Righter, University of Arizona Press, pp. 179-196, 2000. 
[46] G. Robuchon, G. Choblet, G. Tobie, O. Cadek, C. Sotin, O. Grasset, Coupling of thermal evolution and despinning of early Iapetus, Icarus 207 (2010), 959-971.

[47] G. Robuchon, G. Tobie, G. Choblet, O. Čadek, A. Mocquet, Thermal evolution of Mercury: implication for despinning and contraction, 40th Lunar and Planetary Science Conference (2009, The Woodlands, Texas), id. 1866 .

[48] Ch.S. Rodrigues, A.P.S. de Moura, C. Grebogi, Emerging attractors and the transition from dissipative to conservative dynamics, Phys. Rev. E 80 (2009), no. 2, 026205, 8 pp.

[49] Ch.J. Seeton, Viscosity-temperature correlation for liquids, Tribology Lett. 22 (2006), no. 1, 67-78.

[50] A.V. Shatina, Evolution of the motion of a viscoelastic sphere in a central Newtonian field, Cosmic Res. 39 (2001), no. 3, 282-294.

[51] C.K. Shearer et al., Thermal and Magmatic Evolution of the Moon, Rev. Mineral. Geochem. 60 (2006), $365-518$.

[52] G.A. Snyder, L.A. Taylor, C.R. Neal, A chemical model for generating the sources of mare basalts: combined equilibrium and fractional crystalization of the lunar magmasphere, Geochim. Cosmochim. Acta 56 (1992), 3809-3823.

[53] S.C. Solomon et al., The MESSENGER mission to Mercury: scientific objectives and implementation, Planet. Space Sci. 49 (2001) 1445-1465.

[54] T. Spohn, F. Sohl, K. Wieczerkowski, V.Conzelmann, The interior structure of Mercury: what we know, what we expect from Bepi-Colombo, Planet. Space Sci. 49 (2001) 1461-1470.

[55] D.L. Turcotte, On the thermal evolution of the Earth, Earth Planet. Sci. Lett. 48 (1980), 53-58.

[56] R.E. Walpole, R.H. Myers and S.L. Myers, Probability and Statistics for Engineers and Scientists, Prentice Hall, New Jersey, US, 1998.

[57] P. Warren, The lunar magma ocean concept and lunar evolution, Ann. Rev. Earth. Planet. Sci. 13, (1985), 201-240.

[58] J.G. Williams, X.X. Newhall, J. O. Dickey, Lunar moments, tides, orientation, and coordinate frames, Plan. Space Sci. 44 (1996), 1077-1080.

[59] M.M. Woolfson, The origin and evolution of solar system, Graduate series in Astronomy, Institute of Physics Publishing, Bristol, 2000.

[60] M.M. Woolfson, On the origin of planets, Imperial College Press, London, 2011.

[61] C.Z. Zhang, Love numbers of the Moon and of the terrestrial planets, Earth, Moon and Plan. 56 (1992), 193-207. 